\title{
High-Temperature Requirement A 1 Causes Photoreceptor Cell Death in Zebrafish Disease Models
}

\author{
Yoshihito Oura, ${ }^{*}$ Machiko Nakamura, ${ }^{\dagger}$ Tohru Takigawa, ${ }^{*}$ Yoko Fukushima, ${ }^{*}$ Taku Wakabayashi, ${ }^{*}$ Motokazu Tsujikawa, ${ }^{*}$ and \\ Kohji Nishida*
}

From the Department of Ophthalmology, ${ }^{*}$ Osaka University Medical School, Suita; and the Pain and Neuroscience Laboratories, ${ }^{\dagger}$ Daiichi Sankyo Co, Ltd, Tokyo, Japan

\author{
Accepted for publication \\ August 23, 2018. \\ Address correspondence to \\ Motokazu Tsujikawa, M.D., \\ Ph.D., Division of Health Sci- \\ ences, Department of Biomed- \\ ical Informatics, Osaka \\ University Graduate School of \\ Medicine, 1-7 Yamadaoka, \\ Suita, Osaka 565-0871, Jap- \\ an. E-mail: moto@sahs.med. \\ osaka-u.ac.jp.
}

\begin{abstract}
Age-related macular degeneration (AMD) is an important cause of blindness. It is characterized by a retinal pigment epithelium (RPE) disorder that leads to death of photoreceptor cells (PRCS). AMD has a strong genetic association with high-temperature requirement A 1 (HTRA1). The relationship between HTRA1 and the AMD phenotype is unknown. In this study, we show that the expression of HTRA1 in PRCs, as well as in RPE, is increased by the disease-associated HTRA1 mutation and aging. Terminal deoxynucleotidyl transferase-mediated dUTP nick-end labeling assay and quantitative PCR of apoptosisassociated caspases confirmed that PRC-specific overexpression of HTRA1 induced PRC death. Transgenic zebrafish overexpressing human HTRA1 in rod PRCs showed morphologic changes of the RPE, including PRC death and lipofuscin accumulation, features similar to those of early AMD. htra1 expression was also increased in a retinitis pigmentosa zebrafish model compared with wild type. In both fish lines, PRC death was rescued by the suppression of $h$ tra1 by the inhibitor 6 -boroV. AKT-forkhead box 03 signaling downstream of HTRA1 was activated via a tumor growth factor $\beta$ signal, resulting in PRC death. These findings suggest that HTRA1 derived from PRCs is associated with early AMD via PRC death. HTRA1 is a potentially effective target for neuroprotective therapy of early AMD and other degenerative diseases of PRCs. (Am J Pathol 2018, 188: 2729-2744; https://doi.org/10.1016/j.ajpath.2018.08.012)
\end{abstract}

Age-related macular degeneration (AMD) causes visual obstruction attributable to degeneration of the retinal pigment epithelium (RPE), leading to the secondary death of photoreceptor cells (PRCs). ${ }^{1}$ In developed countries with good medical services, AMD mainly causes severe visual impairment with aging. ${ }^{2}$ AMD is classified into two groups: wet AMD and dry AMD (geographic atrophy). ${ }^{3,4}$ Both types have RPE degeneration as a prominent feature, and PRC dysfunction is thought to be a secondary change to RPE degeneration..$^{5-7}$ In wet AMD, abnormal choroidal neovascularization (CNV) secondary to RPE degeneration occurs in the macular region. Repeated hemorrhages and fibrosis from CNV destroy the normal retinal structure and lead to central blindness. ${ }^{8-10}$ Although it had long been a critical issue in the management of wet AMD to suppress $\mathrm{CNV}$, anti-vascular endothelial growth factor treatment has been established as a standard therapy and has improved the visual prognosis of patients with wet AMD. ${ }^{11,12}$ However, visual function recovers only to a limited extent after the development xof CNV. Earlier and more effective intervention is necessary to prevent blindness caused by AMD.

AMD is associated with many factors, such as aging, ${ }^{13}$ hypertension, ${ }^{14}$ smoking history, ${ }^{13,14}$ dietary intake, ${ }^{14,15}$ oxidative stress, ${ }^{16}$ and genetic background. ${ }^{17,18}$ Genomewide association studies have reported strong genetic

\footnotetext{
Supported by the Grants-in-Aid for Scientific Research from the Japanese Society for the Promotion of Science (JSPS KAKENHI) 17K11448 (M.T.) and by Daiichi Sankyo Co. Ltd (M.T.).

Disclosures: Daiichi Sankyo Co, Ltd, provided funding and supplied high-temperature requirement A 1 inhibitor (6-boroV; DPMFKLboroV). M.N. is an employee of Daiichi Sankyo Co, Ltd.

Current address of M.T., Division of Health Sciences, Department of Biomedical Informatics, Osaka University Graduate School of Medicine, Osaka, Japan.
} 
associations with AMD. ${ }^{19-21}$ It was reported that two genes on chromosome 10q26, age-related maculopathy susceptibility 2 (ARMS2) and high-temperature requirement A 1 (HTRAl), were the strongest loci associated with AMD, comparable to the complement factor $\mathrm{H}(\mathrm{CFH})$ locus on chromosome 1q32, although there was little knowledge as to which gene or genetic variation was functionally relevant to AMD pathology. ${ }^{19,22}$ Among many AMD susceptibility genes, the singlenucleotide polymorphism (SNP) rs11200638 in the HTRAl promoter region is one of the strongest disease-associated SNPs, ${ }^{22,23}$ with increased odds ratios of 1.66 in the heterozygote and 11.14 in the homozygote. ${ }^{22}$ On the other hand, genetic variants responsible for disease susceptibility are closer to ARMS2 than to HTRA1 at the $10 \mathrm{q} 26$ locus. ${ }^{24}$

HTRAl is related to the pathogenesis of other diseases, such as cerebral autosomal recessive arteriopathy with subcortical infarcts; leukoencephalopathy, an inherited small-vessel disease $\mathrm{e}^{25}$; rheumatoid arthritis and osteoarthritis $^{26,27}$; and cancer. ${ }^{28}$ HTRAl transgenically overexpressed in mouse RPE induced CNV such as that seen in wet AMD. ${ }^{29}$ It remains unclear, however, how physiological expression of HTRAl causes the retinal phenotype.

We report herein that HTRAl is mainly associated with maintenance of PRCs, not with maintenance of the RPE, and that HTRA1 derived from PRCs induces apoptosis of PRCs and morphologic changes similar to those of early AMD. These findings indicate that AMD is initially a disease of PRCs, which produce more HTRA1, resulting in prepathologic changes of $\mathrm{AMD}$, leading to degeneration of the RPE. Therefore, it is suggested that HTRAl is a potentially effective target for neuroprotective therapy for early AMD and other degenerative diseases of PRCs.

\section{Materials and Methods}

\section{Human Donor Eye}

The use of all human samples in this study adhered to the tenets of the Declaration of Helsinki. A whole eye globe from a73-year-old female cadaver human donor was obtained from SightLife (Seattle, WA). Informed consent for donation of the eye to research was obtained by SightLife. The eye globe was fixed in $4 \%$ (w/v) paraformaldehyde for 24 hours at $4^{\circ} \mathrm{C}$, then used for immunohistochemistry.

\section{Animal Strains}

Zebrafish (Danio rerio) strains were maintained and bred, as previously described. ${ }^{30,31}$ The following zebrafish strains were used: wild-type (WT) AB (Zebrafish International Resource Center, University of Oregon, Eugene, OR), Tg(rho:hsa.RHO_Q344ter), and Tg(rho:hsa.HTRAl). The tol2 transposon system was used to produce $\mathrm{Tg}$ (rho:hsa.HTRA1). rho means driven by a rhodopsin promoter of zebrafish. Tg(rho:hsa.RHO_Q344ter) is a transgenic fish model of autosomal dominant retinitis pigmentosa (RP) expressing human rhodopsin with a carboxyl-terminal chain terminating mutation. ${ }^{32} \mathrm{Tg}($ rho:EGFP $)$, which has a fluorescent rod PRC labeled by enhanced green fluorescent protein (EGFP), was a gift from Dr. Shoji Kawamura (The University of Tokyo, Tokyo, Japan) ${ }^{33}$ and was interbred with other transgenic fish to visualize the PRCs.

For quantitative evaluation of eye-specific expression of target genes at the mRNA and protein levels, enucleated eyes of 20 to 30 fish littermates at 5 days post-fertilization (dpf) were considered as coming from one individual $(N=1)$, because the sample from one fish embryo was too small to analyze. In the sections below, $n$ is used to indicate the number of treated eyes.

Mice (C57BL/6J-Aged), ages 8 and 68 weeks, were obtained from Charles River Laboratories Japan (Yokohama, Japan) and sacrificed a few days after purchase for immunohistochemistry.

\section{Morpholino Knockdown}

Morpholino oligonucleotide knockdowns were performed, as described previously. ${ }^{31}$ mRNA was transcribed with the mMessage Machine kit (Ambion, Thermo Fisher Scientific, Waltham, MA). The concentration of morpholinos used in the experiments was $380 \mathrm{mmol} / \mathrm{L}$. Morpholinos were injected into the embryos within 30 minutes after fertilization, and the embryos were sacrificed at $5 \mathrm{dpf}$. Morpholinos targeted to a splice site of the htrala gene (SP1 and SP2 morpholinos) were designed. These morpholinos block the splicing, and the efficiency of the SP morpholino knockdown was determined by RT-PCR analysis. Morpholino oligonucleotides were ordered from Gene Tools (Philomath, OR) large latent complex. The following morpholinos were used: htrala (SP1), 5' TGTGAGAAACTTACTTGCGGTAAAG-3'; htrAala (SP2), 5'-TGTCTTGTACCTCTGGCCAGTCTGTC-3'; and standard control morpholino, 5'-CCTCTTACCTCAGTTACAATTTATA-3'. The efficacy of SP1 and SP2 morpholinos of htrala was confirmed, and an SP1 morpholino in the knockdown test was used.

\section{Injection of Inhibitor}

HTRA1 inhibitor (6-boroV; DPMFKLboroV) ${ }^{34}$ was provided by Daiichi Sankyo Co, Ltd (Tokyo, Japan). 6-boroV was diluted to 30,100 , and $300 \mathrm{mmol} / \mathrm{L}$ with dimethyl sulfoxide. The control reagent was dimethyl sulfoxide without 6-boroV. Coordinated inhibitor solution was injected into the abdominal yolk sacs of 5-dpf embryos. The treated embryos were sacrificed 24 hours after injection.

\section{Immunohistochemistry}

The mice were anesthetized in $0.1 \%$ tricaine and sacrificed. The eyes were enucleated and fixed with modified Davidson fluid. ${ }^{35}$ After 24 hours, the fluid was exchanged with $20 \%$ glucose aqueous solution and the eyes were kept at $4^{\circ} \mathrm{C}$ 
overnight. The human eye globe was also kept in $20 \%$ glucose aqueous solution for 24 hours at $4{ }^{\circ} \mathrm{C}$ after fixation. The zebrafish embryos were anesthetized in $0.08 \%$ tricaine and placed in $4 \%(\mathrm{w} / \mathrm{v})$ paraformaldehyde, $\mathrm{pH} 7.4$, in phosphate-buffered saline (PBS) overnight at $4{ }^{\circ} \mathrm{C}$ and then incubated in $20 \%$ glucose aqueous solution overnight at $4{ }^{\circ} \mathrm{C}$. The fixed mouse eyes, human eye, and fish embryos were embedded in OCT compound (Neg50, Thermo Fisher Scientific; or Tissue-Tek, Sakura Finetek Japan, Chu-o ku, Tokyo, Japan) and quick frozen in liquid nitrogen. When embedding the samples, they were placed along the pupil/ optic nerve axis to be examined under a light microscope. The samples were cut into sections (10 $\mu \mathrm{m}$ thick).

Before the antigen-antibody reaction was performed, antigen retrieval treatment was performed by $0.05 \%$ citraconic anhydride solution (ImmunoSaver Antigen Retriever; Electron Microscopic Sciences, Hatfield, PA) and surfaceactivating agent $(1 \%$ Tween $/ 0.1 \mathrm{~mol} / \mathrm{L}$ PBS; Tween 20 ; Sigma-Aldrich, St. Louis, MO). The samples were incubated for 40 minutes in a $0.05 \%$ citraconic anhydride solution at $98^{\circ} \mathrm{C}$ in a water bath, the samples were washed with $0.1 \mathrm{~mol} / \mathrm{L} \mathrm{PBS}$, and the samples were reacted with $1 \%$ Tween PBS solution for 5 minutes at room temperature. The sections went through a $0.1 \mathrm{~mol} / \mathrm{L} \mathrm{PBS} / 0.005 \%$ Tween rinse cycle and were preincubated for 60 minutes in a solution containing $4 \%$ to $10 \%$ bovine serum albumin. The sections were then incubated in a solution containing $0.1 \mathrm{~mol} / \mathrm{L}$ PBS, $4 \%$ bovine serum albumin, and the primary antibody at $4{ }^{\circ} \mathrm{C}$ overnight. After another $0.1 \mathrm{~mol} / \mathrm{L} \mathrm{PBS} / 0.005 \%$ Tween rinse cycle, the sections were incubated with Alexa Flour 647-conjugated IgG antibodies (1:1000; Jackson ImmunoResearch, West Grove, PA) for HTRA1 staining, Alexa Fluor 594-conjugated IgG antibodies (1:1000; Jackson ImmunoResearch) appropriate for other primary antibodies, and DAPI (1:1000; Cytoskeleton, Denver, CO) for nuclear staining at room temperature for 1 hour; then, they were mounted with aqueous mounting medium (PermaFluor; Thermo Fisher Scientific). The sections were analyzed with the use of the confocal fluorescence laser microscopes LSM 710 (Carl Zeiss, Jena, Thüringen, Germany) and an ELYRA superresolution microscope (Carl Zeiss). The images were processed with Zen (Carl Zeiss) and Photoshop CC 2015 software (Adobe, San Jose, CA). The following primary antibodies and dilutions were used: rabbit polyclonal antiHtrA1 antibody (1:100; Proteintech, Chicago, IL), rabbit monoclonal anti-Akt (pan; C67E7; 1:100; Cell Signaling, Danvers, MA), rabbit monoclonal anti-phosphorylated Akt (p-Akt; Ser473; D9E; 1:100; Cell Signaling), rabbit polyclonal anti-FKHRL1 [forkhead in rhabdomyosarcoma-like 1, former name of forkhead box O (FoxO) 3a; H-144; 1:100; Santa Cruz Biotechnology, Dallas, TX], rabbit polyclonal anti-phosphorylated FoxO3a (Ser318/321; 1:100; Cell Signaling), goat polyclonal anti-latency-associated peptide (LAP) transforming growth factor (TGF)- $\beta$ antibody (1:100; R\&D Systems, Minneapolis, MN), rabbit polyclonal anti-TGF-1 antibody (1:100; Promega, Fitchburg, WI), mouse monoclonal anti-actin (Calbiochem, Merck Millipore, Billerica, MA), and mouse polyclonal anti-human latent TGF- $\beta$ bp1 (LTBP-1; 1:100; R\&D Systems). To visualize the nuclei, DAPI (1:1000; Cytoskeleton) was added to the secondary antibody solution.

\section{Histology}

For histologic analysis of zebrafish, embryos were freeze sectioned, as described above. After the rinse cycle of 0.1 $\mathrm{mol} / \mathrm{L} \mathrm{PBS} / 0.005 \%$ Tween, F-actin and nuclei of the sections were stained by rhodamine phalloidin (1:250; Cytoskeleton) and DAPI (1:1000; Cytoskeleton) for 1 hour at room temperature. After the slides were mounted in aqueous mounting medium, the phenotypes of the embryo were observed and recorded with the use of the AxioVert 200 microscope (Carl Zeiss Japan, Tokyo, Japan). The images were recorded with AxioVision version 4.8 (Carl Zeiss Japan) and processed by Photoshop CC (Adobe). The number of rod PRCs was counted as the number of fluorescence-positive cells in each image.

\section{Apoptosis Assay}

An apoptosis assay was performed with a terminal deoxynucleotidyl transferase-mediated dUTP nick-end labeling (TUNEL) commercial kit (ApopTag Fluorescein in Situ Apoptosis Detection Kit; S7110; Chemicon, Merck Millipore). The sections were stained by DAPI (1:1000; Cytoskeleton) and imaged to count the number of TUNELpositive fluorescent cells in the PRC layer by the same procedure as that used to count EGFP-labeled PRCs.

\section{Real-Time PCR Assay}

For analysis of whole eyes, enucleated eyes from individual fish were immersed in TRIzol Reagent (Thermo Fisher Scientific) after pooling them in RNA stabilization solution (RNAlater Tissue Collection; Thermo Fisher Scientific). For cell samples, cells were directly immersed in TRIzol after collecting them by centrifuge. Isolation of total RNA was performed with an RNeasy Mini Kit (Qiagen, Hilden, Mettmann, Germany). Total RNA was isolated according to the manufacturer's instructions, and RNA was resuspended in $20 \mathrm{~mL}$ of RNase-free water. After the aqueous solution of RNA was treated with DNase, the RNA was extracted with phenol-chloroform. RNA was reverse transcribed with the SuperScript VILO cDNA Synthesis kit (Life Technologies, Thermo Fisher Scientific). The reverse-transcribed mRNA products (cDNA) were amplified and analyzed by real-time quantitative PCR (Applied Biosystems 7500 Fast Real-Time PCR System; Applied Biosystems, Thermo Fisher Scientific) using the TAKARA SYBR Green PCR mixture (Takara Bio Inc., Kusatsu, Shiga, Japan). Each sample was measured in duplicate. The expression of the target genes [htrala, htralb, actin $\beta 1$ (actb1), casp3a, casp3b, casp8, 
Table 1 Primers Used for Quantitative PCR

\begin{tabular}{|c|c|c|}
\hline Gene & Forward primer & Reverse primer \\
\hline casp $3 a$ & $5^{\prime}-$ CAGGACAGGCATGAACCCACGC $-3^{\prime}$ & 5'-GCGGCTGTGATCGTCATGGGC-3' \\
\hline casp3b & 5'-CGCTGCACGTCACTCGTGGG-3' & $5^{\prime}-$ TCCTGGTGCGGTGGAGTAGGC-3' \\
\hline$a c t b 1$ & 5'-GGCCCATCCATCGTTCACAG-3' & $5^{\prime}$-CGAGAGTTTAGGTTGGTCGTTCG-3' \\
\hline htra1a & $5^{\prime}$-ACATCTGCGAACTGAAGCGG-3' & 5'-CCGCTGTATGAAGAGCACCG-3' \\
\hline htra1b & $5^{\prime}$-CACAGGGAAACTGGGAGACC-3' & 5'-TCTCACGGTAGTCGTTGCAC-3' \\
\hline $\operatorname{tgfb3}$ & $5^{\prime}$-GGACCGAGCAGAGAATCGAG-3' & 5'-ACGTCGAAGGAAACCCACTC-3' \\
\hline
\end{tabular}

tgfbla, $t g f b 2$, and $t g f b 3$ ] was normalized by the comparative $\mathrm{C}_{\mathrm{T}}$ method and an internal control (actbl) and calculated by the $\Delta \Delta \mathrm{C}_{\mathrm{T}}$ method. The RT-PCR primers were synthesized from Invitrogen (Thermo Fisher Scientific). The primer sequences are shown in Table 1.

\section{Western Blot Analysis}

Enucleated eyes were pooled from fish embryos in radioimmunoprecipitation lysis buffer containing protease inhibitors (Complete Mini Protease Inhibitor Cocktail;
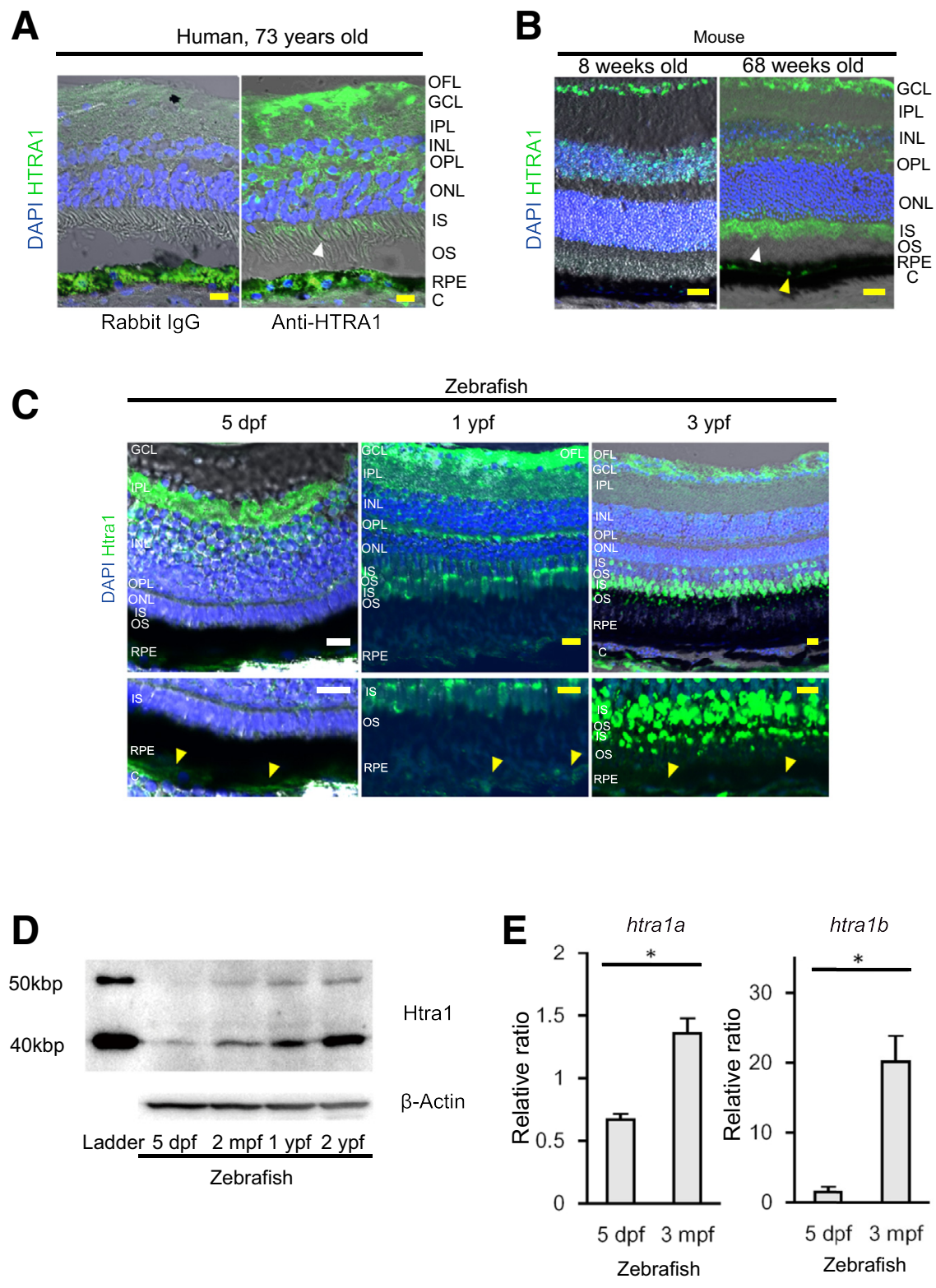

Figure 1 HTRA1, expressed in the inner segment (IS) of the photoreceptor cells and outer plexiform layer (OPL), increases with aging. A-C: Immunohistochemistry of HTRA1 (green) and nuclei (blue; counterstained by DAPI) in retinal sections of human donor eye (A), mouse (B), and zebrafish (C). A: In a 73-year-old female human retinal section, positive stains for HTRA1 are seen in all layers, including the ganglion cell layer $(\mathrm{GCL})$, the inner nuclear layer (INL), the outer nuclear layer $(\mathrm{ONL})$, and the inner segment (white arrowhead) of the photoreceptor cell. The retinal pigment epithelium (RPE) is stained by both antiHTRA1 antibody and rabbit IgG as negative control. B: HTRA1 staining of HTRA1 (green) in the 68-week-old mouse is stronger than that in the 8week-old mouse in the inner segment (white arrowhead) and retinal pigment epithelium (yellow arrowhead). In zebrafish, Htra1 staining (green) of the inner segment and the outer segment (OS; yellow arrowhead) becomes stronger with aging. C: The retinal pigment epithelium (yellow arrowheads) is stained weakly, not strongly as in the mouse. D: Western blot analysis reveals that Htra1 expression increases with age. E: $h$ tra1 $a$ and $h$ tra $1 b$ mRNA expression, normalized to actin cytoplasmic 1 (actb1) and assessed by real-time PCR, indicates an increase in htra1a with age in wild-type zebrafish. Data are expressed as means \pm SEM. $n=4$ (E, $5 \mathrm{dpf}$ and $3 \mathrm{mpf})$. ${ }^{*} P<0.05$ (unpaired $t$-test). Scale bars: $20 \mu \mathrm{m}$ (A-C, yellow); $10 \mu \mathrm{m}$ (C, white). C, choroid; $\mathrm{dpf}$, days post-fertilization; IPL, inner plexiform layer; mpf, months post-fertilization; $0 \mathrm{FL}$, optic fiber layer; WT, wild-type; ypf, years postfertilization. 


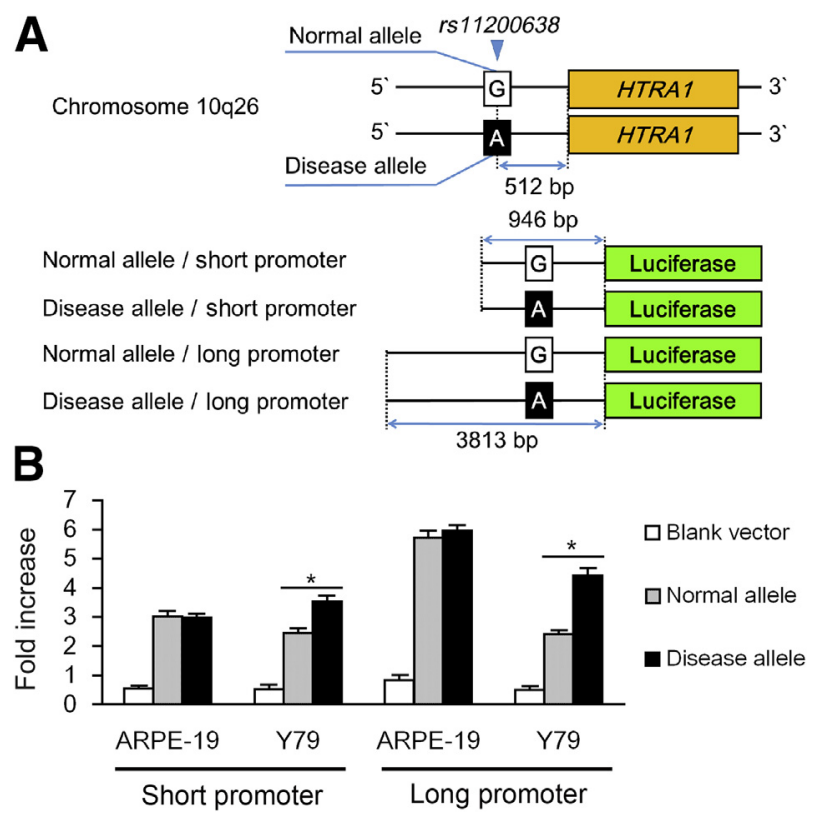

Figure 2 Representative single-nucleotide polymorphism of HTRA1 promoter (rs11200638) is more associated with photoreceptor cells than with retinal pigment epithelium in vitro. A: Schematic diagram of the normal allele $(\mathrm{G})$ /disease-susceptibility allele $(\mathrm{A})$ inserted into HTRA1 promoter region in the constructs used for luciferase assays (orange region contains the HTRA1 regulatory element; green region contains the luciferase reporter gene). G/A single-nucleotide polymorphisms of $r 11200638$ are located $512 \mathrm{bp}$ from the start point of transcription of HTRA1 in the upstream direction. Four luciferase constructs were generated to analyze HTRA1 transcription regulator activity: normal allele $(\mathrm{G})$ /long promoter, normal allele $(\mathrm{G})$ /short promoter, diseasesusceptibility allele (A)/long promoter, and disease-susceptibility allele (A)/short promoter. B: Luciferase assay of G/A single-nucleotide polymorphism inserted into HTRA1 promoter in ARPE-19 (human retinal pigment epithelium cell line) and Y79 (human rod photoreceptor cell line). Data are expressed as means \pm SEM. $N=3\left(\right.$ B). ${ }^{*} P<0.05$.

Roche, Basel, Switzerland) and phosphatase inhibitor cocktail (PhosSTOP; Roche). The eyes were triturated with a pestle and sonicator and incubated on ice for 30 minutes. The samples were centrifuged at $9100 \times g$ for 12 minutes at $4^{\circ} \mathrm{C}$. The supernatant was collected, electrophoresis was performed, and the proteins were transferred to membranes using the NuPAGE electrophoresis system and the iBlot Dry Blotting System (Invitrogen, Thermo Fisher Scientific). The transferred membranes were incubated with primary antibodies at $4^{\circ} \mathrm{C}$ overnight after being placed in blocking buffer containing $4 \%$ bovine serum albumin for 1 hour at room temperature, and the signal was detected using the appropriate secondary horseradish peroxidase-conjugated antibodies (1:10,000; Jackson ImmunoResearch). The labeled protein bands on the transferred membranes were visualized by chemiluminescence reagents (ECL Prime Western Blot Analysis Detection Reagents; GE Healthcare, Chicago, IL), and images were obtained by ChemiDoc XRS (Bio-Rad Laboratories, Hercules, CA). Each experiment was performed at least twice, and representative data are shown. The following primary antibodies and dilutions were used: rabbit polyclonal anti-HtrA1 antibody (1:1000;
Proteintech), goat polyclonal anti-LAP TGF- $\beta$ antibody (1:1000; R\&D Systems), rabbit polyclonal anti-TGF- $\beta 1$ antibody (1:1000; Promega), rabbit monoclonal anti-Akt (pan; C67E7; 1:1000; Cell Signaling), rabbit monoclonal anti-p-Akt (Ser473; D9E; 1:1000; Cell Signaling), and mouse monoclonal anti-actin (1:10,000; Calbiochem). The actin band was used as a loading control.

\section{In Situ Hybridization}

Eyes from WT mice were removed and immersed in $4 \%$ formaldehyde overnight at $4^{\circ} \mathrm{C}$. The eyes were then embedded in paraffin and divided into sections (10 $\mu \mathrm{m}$ thick). HTRAl cDNA was PCR amplified with the following primers: forward primer, 5'-GGATCCGAATGACCCTTCGCTGGGCC-3'; and reverse primer, 5'-CTCGAGCCACAAGCTTGGTGGACAGTG-3'. Digoxigenin (DIG)-labeled antisense/sense probes were used for in situ hybridization. To make DIG-labeled probes, HTRAl cDNA was PCR amplified and subcloned into the pBluescript SK vector (Stratagene, Agilent Technologies, Santa Clara, CA). The DIG-labeled antisense and sense probes were transcribed with a DIG RNA Labeling kit (SP6/T7; catalog number 11 175025 910; Merck KGaA, Darmstadt, Germany). The labeled product was fragmented, lysed by incubation in hydrolysis buffer for 85 minutes at $60^{\circ} \mathrm{C}$, precipitated in $\mathrm{NH}_{4} \mathrm{AC}$ and ethanol, and dissolved in dithiothreitol. After prehybridization at $65^{\circ} \mathrm{C}$ in hybridization buffer containing $50 \%(\mathrm{w} / \mathrm{v})$ formamide, $4 \times$ saline sodium citrate (SSC), $1 \times$ Denhardt's solution, $10 \%(\mathrm{w} / \mathrm{v})$ dextran sulfate, and 0.4 $\mathrm{mg} / \mathrm{mL}$ salmon sperm DNA (Stratagene) for 30 minutes, the sections were washed in $4 \times$ SSC for 5 minutes and hybridized overnight at $65^{\circ} \mathrm{C}$ in hybridization buffer containing $1 \times 10^{7} \mathrm{cpm} / \mathrm{mL}$ of labeled probe. The sections were then washed in $4 \times$ SSC containing $50 \%(\mathrm{w} / \mathrm{v})$ formamide for 30 minutes, $2 \times$ SSC containing $50 \%(\mathrm{w} / \mathrm{v})$ formamide for 30 minutes, $1 \times \mathrm{SSC}$ containing $50 \%(\mathrm{w} / \mathrm{v})$ formamide for 45 minutes, and $1 \times$ SSC for 10 minutes. The presence of mRNA in paraffin section tissue was detected with nitro blue tetrazolium/5-bromo-4-chloro-3-indolyl-phosphate through an alkaline phosphatase-conjugated anti-DIG antibody (Roche Diagnostics, Rotkreuz, Switzerland). The slide images were acquired with a Zeiss microscope equipped with a chargecoupled device camera (AxioCam ERc 5s; Carl Zeiss Japan).

\section{Transmission Electron Microscopy}

The zebrafish embryos were fixed in $2.5 \%$ glutaraldehyde and $2 \%$ paraformaldehyde in $0.1 \mathrm{~mol} / \mathrm{L}$ PBS for 2 hours. The eyeballs were washed overnight at $4^{\circ} \mathrm{C}$ in the same buffer and post-fixed for 2 hours with aqueous osmium tetroxide in $0.1 \mathrm{~mol} / \mathrm{L} \mathrm{PBS}$. The samples were dehydrated through a graded ethanol series and embedded in Epon 812 (TAAB Laboratories Equipment, Aldermaston, UK). Ultrathin sections (70 nm thick) were collected on copper grids, double stained with uranyl acetate and lead citrate, 

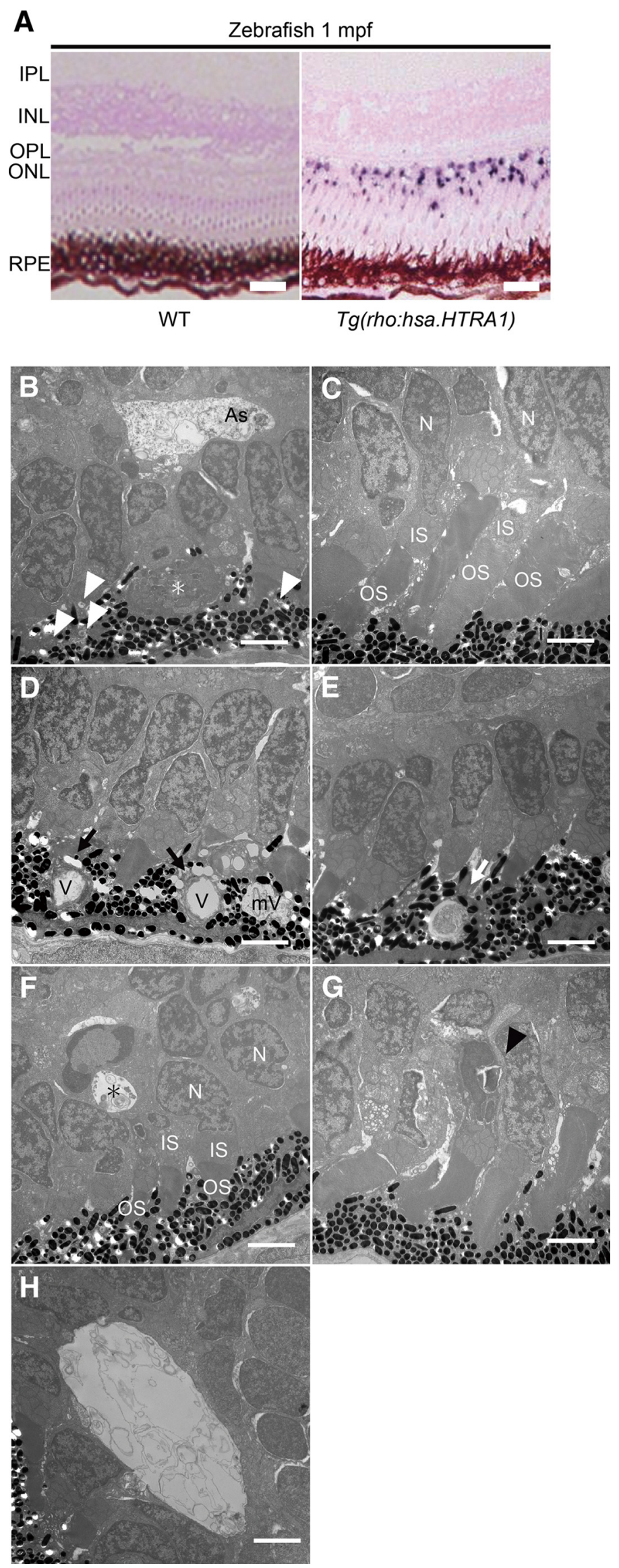

Figure 3 In situ hybridization and transmission electron micrographs of wild-type (WT) zebrafish and $\mathrm{Tg}$ (rho:hsa.HTRA1) at 1 month post-fertilization (mpf). A: In situ hybridization for human HTRA1 and zebrafish htra1a on paraffin retinal sections of WT and $T g$ (rho:hsa.HTRA1) at $1 \mathrm{mpf}$. An mRNA probe was constructed to recognize the common region between human HTRA1 and zebrafish htra1a. Purple represents expression of human HTRA1 or zebrafish htra1a mRNA. B-H: Ultrastructure of the retina of $T g$ (rho:hsa.HTRA1) and WT 1-year postfertilization fish by transmission electron microscopy. B: Migrated astrocyte in the outer nuclear layer (ONL) and superficial deposits of lipofuscin; a large aggregate (white asterisk) and several small granules (white arrowheads) are shown between the outer segment (OS) of the photoreceptor cells and the retinal pigment epithelium (RPE). C: Normal photoreceptor layer with dense nuclei and moderately rounded outer segments. D: Somatic vesicles of the retinal pigment epithelium. Note the round blank vesicles and misshapen vesicles (mVs) containing lysates. E: Aggregate of lipofuscins, including undigested disk in the retinal pigment epithelium (white arrow). F: Abnormal photoreceptor cell with an extracellular vesicle (black asterisk) and thin, shortened outer segments. G: Condensed nuclei of photoreceptor cell (black arrowhead). H: Huge vacuole in the outer nuclear layer. Scale bars: $10 \mu \mathrm{m}(\mathbf{A}) ; 1 \mu \mathrm{m}(\mathbf{B}-\mathbf{H})$. As, astrocyte; $\mathrm{INL}$, inner nuclear layer; IPL, inner plexiform layer; $\mathrm{IS}$, inner segment; $\mathrm{N}$, nucleus; $\mathrm{OPL}$, outer plexiform layer; $\mathrm{V}$, vesicle. 
and then examined with an H-7100 transmission electron microscope (Hitachi, Tokyo, Japan).

\section{Cell Culture}

ARPE-19 cells of a human retinal pigment epithelial cell line were cultured in Dulbecco's modified Eagle's medium/ Ham's F12 medium supplemented with $1 \%$ v/v fetal bovine serum. When cell confluency reached $80 \%$ on a $10-\mathrm{cm}$ dish, the cells were detached with $0.25 \% \mathrm{v} / \mathrm{v}$ trypsin-EDTA in PBS. Y79 cells were maintained by suspension culture of RPMI 1640 medium with L-glutamate and $10 \% \mathrm{v} / \mathrm{v}$ fetal bovine serum in 10-cm dishes. At approximately every 3 to 4 days, the cells were harvested and $5.0 \times 10^{5}$ cells were seeded in fresh $10-\mathrm{mL}$ medium.

\section{Luciferase Assay}

Luciferase assay was performed with a Ready-To-Grow Dual Secreted Reporter Vector Kit (631735; Clontech, Mountain View, CA), according to the manufacturer's instructions. Two different lengths of promoter regions (946-bp short promoter and 3813-bp long promoter) of HTRA1, including rs 11200638, were cloned. The regions were recovered from genomic PCR, cloned into pMetLuc Reporter Vector (Clontech), and genotyped by direct sequence. These luciferase constructs were transfected into ARPE-19 and Y79 cells using Lipofectamine 3000 (L3000015; Invitrogen), and the signals were measured 4 days after transfection. Luciferase assay was performed with Ready-To-Glow Secreted Luciferase Reporter Systems (631726; Clontech). The transcriptional activity was measured using a secreted embryonic alkaline phosphatase, cloned into phosphorylated secreted embryonic alkaline phosphatase 2 control vector, including the kit. Luciferase activity was calculated from the Metridia luciferase activity divided by the secreted embryonic alkaline phosphatase activity.

\section{Measurement of Autofluorescence}

Eyes from fish [WT and $T g$ (rho:hsa.HTRAl) at 3 months post-fertilization (mpf)] and mice (C57BL/6 at 1 year) were fixed, mounted, and divided into sections using the same procedure as that used for immunohistochemistry. The sections were excited by light at $488 \mathrm{~nm}$, and autofluorescence was detected with a 562- to 602-nm bandpass filter under a confocal microscope (LSM710 AxioObserver; Zeiss). The high-contrast images between lipofuscin and other retinal deposits were captured among a broad emission of many retinal deposits ( 500 to $800 \mathrm{~nm}$ ) by using a narrow spectral window. ${ }^{36,37}$

\section{Statistical Analysis}

The statistical significance of the results was determined by unpaired $t$-tests. Statistical analysis was performed with JMP software version 10 (SAS Japan Institute, Osaka,
Japan). $P<0.05$ was considered to indicate statistical significance. Results are presented as means $\pm \mathrm{SD}$, except for RT-PCR results, which are presented as means \pm SEM.

\section{GenBank Accession Numbers}

DNA sequence information in this study is available through GenBank (https://www.ncbi.nlm.nih.gov/genbank) accession numbers, as follows: HTRAl (human), NM 000539; htrala (zebrafish), NM_001002219.1; htralb (zebrafish), NM_001111182.1; actb1 (zebrafish), NM_ 131031.1; casp3a (zebrafish), NM_131877.2; casp3b (zebrafish), NM_001048066.1; casp8 (zebrafish), NM_ 131510.2; tgfbla (zebrafish), NM_182873.1; tgfb2 (zebrafish), NM_194385.1; and tgfb3 (zebrafish), NM_ 194386.2.

\section{Approval of the Study}

The WT mice (C57BL/6J-Aged) were housed at $25^{\circ} \mathrm{C}$ on a 12-hour light/12-hour dark cycle (with light $<10$ lux) and were fed standard rodent chow and water ad libitum. All animal experiments were performed under approved protocols and in accordance with the recommendations for the proper care and use of laboratory animals. The experimental protocols were approved by the Osaka University Animal Ethics Committee in compliance with The Association for Research in Vision and Ophthalmology statement for the use of animals in vision research.

\section{Results}

HTRA1 Is Expressed in All the Layers that Include PRCs

The endogenous expression pattern of HTRA1 was first examined in a 73-year-old female human donor eye by immunohistochemistry (Figure 1A). Diffuse staining was found in almost all the retinal layers. The inner segments of the PRCs were stained diffusely (Figure 1A). RPE was stained by both anti-HTRA1 antibody and rabbit IgG as a negative control, but it showed slightly stronger staining by anti-HTRA1 antibody than by rabbit IgG. The specificity of anti-HTRA1 antibody was confirmed in staining of Tg(rho:hsa.HTRAl) fish, which expressed human HTRA1 in PRCs (Supplemental Figure S1). Next, the endogenous expression pattern of HTRA1 was examined in 8- and 68week-old mice, and the difference in HTRA1 expression between young and old mice was studied (Figure 1B). Strong staining was detected in the ganglion cell layer of both 8- and 68-week-old mice, and no difference was found between age. In the inner plexiform layer, outer plexiform layer, outer nuclear layer, and RPE, no signals were detected in 8-week-old mice, but unequal diffuse staining was detected in the inner plexiform layer and the outer plexiform layer in 68-week-old mice, and recognizable granular staining was also detected in the outer nuclear 

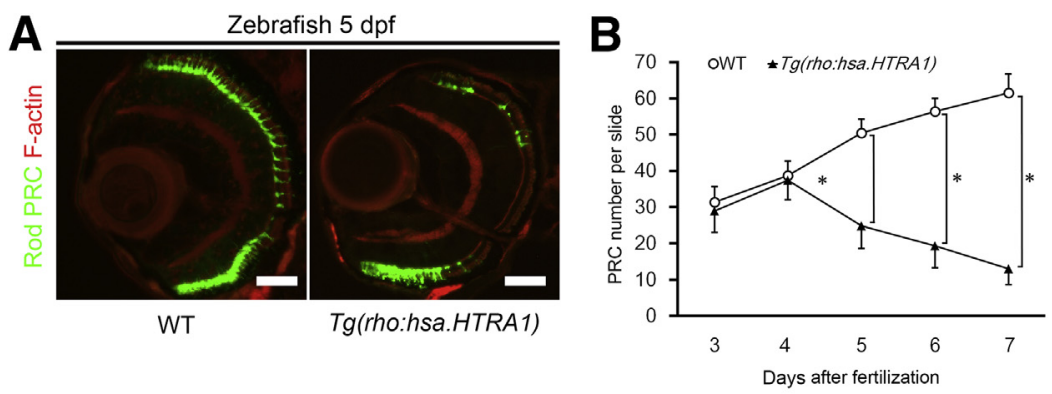

$\mathbf{E}$
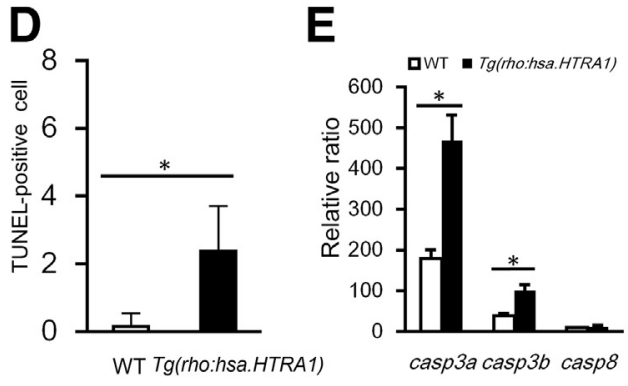

$\mathbf{F}$

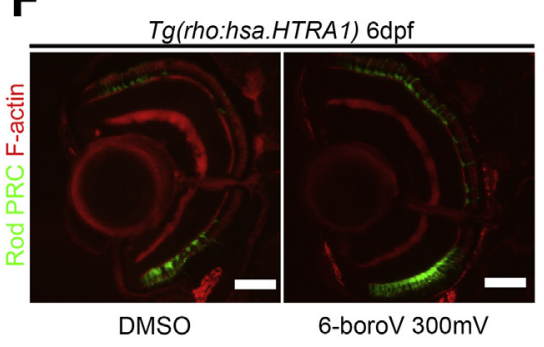

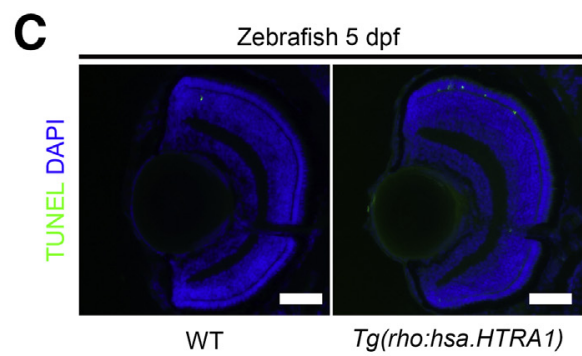

G

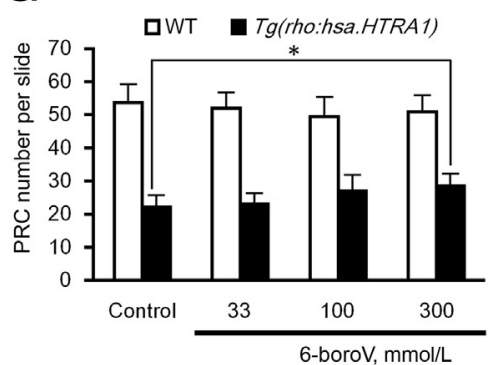

Figure 4 Increased HTRA1 induces apoptosis in photoreceptor cells. A: Representative images in whole eye sections of wild-type (WT) and Tg(rho:hsa.HTRA1) fish at 6 days post-fertilization (dpf). Photoreceptor cells are visualized with enhanced green fluorescent protein (EGFP; green), and F-actin is visualized with phalloidin (red). B: The number of rod photoreceptor cells of WT (vertical axis) and Tg(rho:hsa.HTRA1) fish at 3, 4, 5, 6, and 7 dpf (horizontal axis). Note that rod photoreceptor cells start to decrease at $5 \mathrm{dpf}$. C: Representative images of terminal deoxynucleotidyl transferase-mediated dUTP nick-end labeling (TUNEL) assay in whole eye sections of WT and rho::HTRA1 fish at $5 \mathrm{dpf}$. Note that a few TUNEL-positive cells (green) are detectable only in Tg(rho:hsa.HTRA1). Nuclei are visualized with DAPI (blue). D: Number of TUNEL-positive cells of WT and $\operatorname{Tg}($ rho:hsa.HTRA1) fish at 5 dpf. E: Expression of casp3a, casp3b, and casp8 mRNA in WT and Tg(rho:hsa.HTRA1) fish at $5 \mathrm{dpf}$. Data are normalized to actin cytoplasmic 1 (actb1). F: Representative images of whole eye sections of $\mathrm{Tg}$ (rho:hsa.HTRA1) 24 hours after injection of dimethyl sulfoxide (DMSO; control) and 6-boroV (HTRA1 inhibitor) at 5 dpf. Rod photoreceptor cells are visualized with EGFP (green), and F-actin is visualized with phalloidin (red). G: Graph of the number of rod photoreceptor cells of Tg(rho:hsa.HTRA1) 24 hours after injection with control (DMSO) and 6-boroV (HTRA1 inhibitor) at the following concentrations: control (DMSO), 33, 100, and $300 \mathrm{mmol} / \mathrm{L}$. Data are expressed as means \pm SD (B, D, and $\mathbf{G})$ or means \pm SEM $(\mathbf{E}) . n=8$ per group $(\mathbf{B}, \mathbf{D}$, and $\mathbf{G}) ; n=4$ per group $(\mathbf{E})$. ${ }^{\star} P<0.05$. Scale bar $=10 \mu \mathrm{m}(\mathbf{A}, \mathbf{C}$, and $\mathbf{F})$. PRC, photoreceptor cell.

layer and the RPE (Figure 1B). The most significant changes of staining were found in the inner segment of the PRCs of all the retinal layers (Figure 1B). These results showed that all the retinal layers, especially in PRCs, expressed HTRA1, and some retinal layers that include PRCs exhibited significant changes with aging.

\section{Htra1 Expression Increases with Age}

The time course of expression of zebrafish htra1 in the WT zebrafish retina was studied by immunohistochemistry at the following stages: $5 \mathrm{dpf}, 1$ year post-fertilization (ypf), and 3 ypf (Figure 1C). In the outer segment and inner segments of PRCs, staining was stronger with age. In the larvae at $5 \mathrm{dpf}$, htra1-positive cells were almost undetectable in PRCs (Figure 1C), but htral expression could be detected in the outer nuclear layer and outer segments of the PRCs in 1- and 3-ypf fish (Figure 1C). The RPEs were stained weakly and showed no significant difference in strength of the signals among them (Figure 1C). The protein level of htra1 increased with age by immunoblotting whole lysates of zebrafish eyes, consistent with the immunohistochemical results (Figure 1D). Two paralogs of htral (htrala/htralb) had been reported in zebrafish, ${ }^{38,39}$ and htrala (alias zHtrAl) had been used in terms of $90 \%$ sequence identity in the protease domain with HTRAl. ${ }^{38}$ The mRNA levels of htrala and htralb in whole eyes significantly increased by 2- and 13.5-fold, respectively, from $5 \mathrm{dpf}$ to $3 \mathrm{mpf}$ in the whole eye of WT zebrafish (Figure 1E): htra1a, $0.67 \pm 0.07$ $(5 \mathrm{dpf})$ versus $1.36 \pm 0.26(3 \mathrm{mpf})(P<0.0001)$; and htra1b, $1.49 \pm 1.18(5 \mathrm{dpf})$ versus $20.20 \pm 8.46(3 \mathrm{mpf})$ $(P=0.0003)$. These results showed that expression of htral in the eye, especially in PRCs, increased with age.

\section{An SNP of HTRA1 Promoter (rs11200638) Is More Functionally Associated with PRCs}

The age-related increase of htral expression in PRCs of zebrafish suggests that HTRAl has a biological effect not only on the RPE but also on the PRCs under pathologic conditions, such as aging. Therefore, the effect of a reported SNP was investigated in PRCs and the RPE in a human cohort genome-wide association study. ${ }^{22}$ The SNP (rs11200638) of the HTRAl promoter is an important risk factor intimately connected with $\mathrm{AMD}$ and $\mathrm{CNV} .{ }^{40-42}$ Using reporter assays in vitro, the promoter activity of 


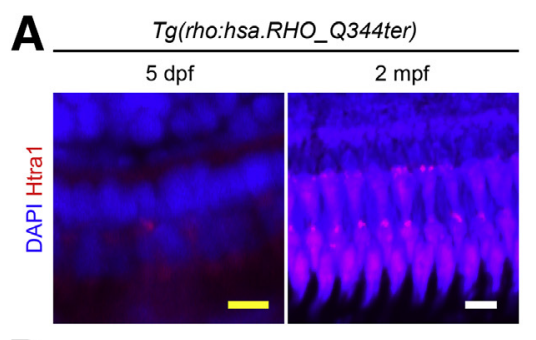

D

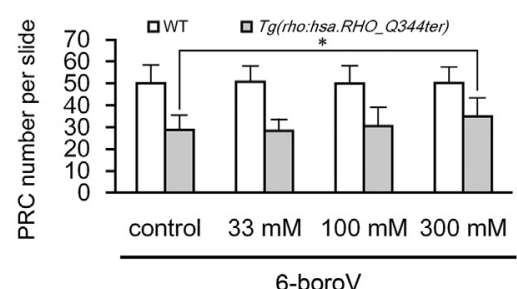

B
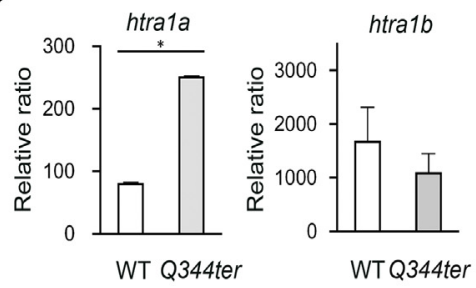

E

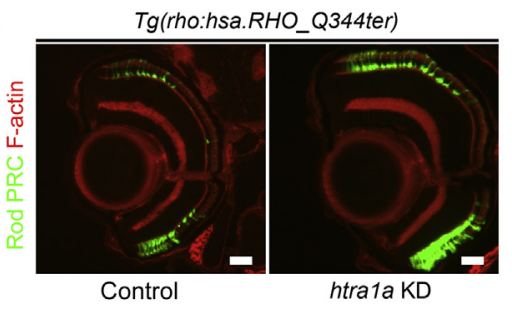

C

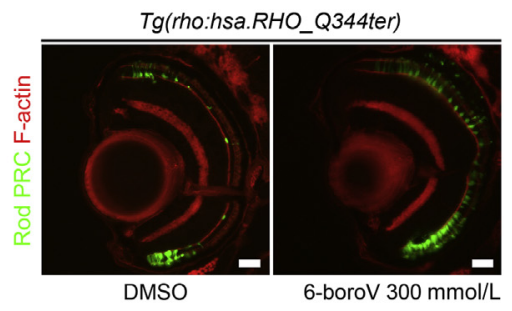

$\mathbf{F}$

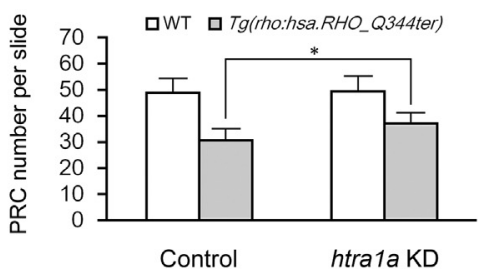

Figure 5 Prevention of overexpressed HTRA1/endogenous htra1a restores photoreceptor cells in Tg(rho:hsa.HTRA1) and Tg(rho:hsa.RHO_Q344ter). A: Immunohistochemistry of retinal sections of $\mathrm{Tg}$ (rho:hsa.RHO_Q344ter) at 5 days post-fertilization (dpf) and 2 months post-fertilization (mpf; red, HTRA1; blue, nuclei counterstained by DAPI). B: Expression of $h$ tra1a and $h$ tra1b mRNA in whole eyes of wild-type (WT) and Tg(rho:hsa.RHO_Q344ter) (Q344ter) zebrafish at 5 dpf. Data are normalized to actb1. C: Representative images of whole eye sections of $T g$ (rho:hsa.RHO_Q344ter) 24 hours after injection of dimethyl sulfoxide

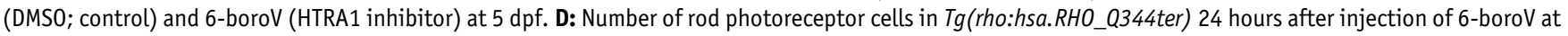
the following concentrations: DMSO (control), 33, 100, and $300 \mathrm{mmol} / \mathrm{L}$. E: Representative images of whole eye sections of Tg(rho:hsa.RHO_Q344ter) 5 days after injection of control morpholino oligonucleotides and morpholino antisense oligonucleotides of $h$ tra1 $a$ in embryos after fertilization. F: Comparison between the number of rod photoreceptor cells in WT and rho::RHOQ344ter in E. C and E: HtrA1 rod photoreceptor cells are visualized with enhanced green fluorescent protein (green), and F-actin is visualized with phalloidin (red). Data are expressed as means \pm SEM (B) or means \pm SD (D and F). $n=6$ to 12 per group (B, D, and $\mathbf{F}$ ). ${ }^{*} P<0.05$ (unpaired $t$-test). Scale bars: $5 \mu \mathrm{m}$ (A, yellow); $10 \mu \mathrm{m}(\mathbf{A}, \mathbf{C}$, and $\mathbf{E}$, white). htra1a KD, knockdown of htra1a; PRC, photoreceptor cell.

HTRA1 was examined in both a human RPE cell line (ARPE-19) and a human retinoblastoma cell line, which expresses rod-specific genes (Y79). The secreted embryonic alkaline phosphatase expression control vector, phosphorylated secreted embryonic alkaline phosphatase 2, was cotransfected to control transfection efficacy. HTRAl promoter regions, including either a normal allele $(\mathrm{G})$ or a disease-susceptibility allele (A), were recovered from human DNA and cloned into a luciferase vector (Figure 2A). The precise area of the HTRAl promoter is unclear, although the SNP (rs11200638) is located $512 \mathrm{bp}$ forward from the transcriptional start site of the HTRAl gene. Two different lengths of the HTRAl promoter for a normal allele $(\mathrm{G})$ and a disease-susceptibility allele (A) were used to check whether the transcriptional activity depended on promoter length. Reporter genes constructed with different lengths of the HTRAl promoter expressed both ARPE-19 and Y79. In both regions of the reporter, the effect of the disease-susceptibility allele (A) was much more apparent in the PRC-like cell line than in the RPE cell line (Figure 2B). In Y79, the activity of both short and long HTRA1 promoters with the disease-susceptibility allele (A) was 1.42 and 1.83 times greater, respectively, than that of the HTRA1 promoter with a normal allele (G). In ARPE-19, there was no significant difference between them, with expression ratios for normal alleles (A)/disease alleles $(\mathrm{G})$ of 0.98 (short promoter) and 1.03 (long promoter).

These findings suggest that HTRAl may be more expressed in PRCs than in the RPE under a genetic background.

\section{Aged $\operatorname{Tg}$ (rho:hsa.HTRA1) Fish Show Characteristics of Early AMD}

Two important risk factors for AMD are aging and genetic factors that increase the expression of HTRAl in PRCs. It was then investigated whether overexpression of HTRAl in PRCs can cause the AMD phenotype. A zebrafish line overexpressing HTRAl [Tg(rho:hsa.HTRA1)] in rod PRCs was generated using a previously reported system. ${ }^{32}$ The transgene was specifically expressed in rod PRCs, as intended, as confirmed by in situ hybridization of Tg(rho:hsa.HTRAl) in 1-mpf fish (Figure 3A). The ultrastructure of the retina of $T g$ (rho:hsa.HTRAl) and WT 1-ypf fish was examined by transmission electron microscopy (Figure 3, B-H).

The most important finding was the presence of a sedimentary layer composed of more granules of lipofuscin and melanolipofuscin between the outer segmental layer of the PRCs and the apical side of the RPE (Figure 3B), although basal laminar deposits and accumulation of lipofuscin were not detectable on the basal side of the RPE. In WT retina, there was no deposition of lipofuscin and melanolipofuscin (Figure 3C). Moreover, migration of astrocytes to damaged areas of the outer neural retina was observed in $T g(r h o: h$ sa.HTRA1) (Figure 3B), which is also seen in the early AMD retina. The RPE itself had pathologic changes involving some vacuoles and necrotic astrocytes similar to those in PRCs of $T g$ (rho:hsa.HTRAl) (Figure 3D). A small vacuole containing undigested disks and a drusen located internally at the layer of the RPE and not at the basal side of 
the RPE was also observed (Figure 3E). The PRCs themselves also showed strong abnormalities. The outer segments of the PRCs in WT fish were of the same length and arranged in order (Figure 3C), but those of $\mathrm{Tg}$ (rho:hsa.HTRA1) fish were crooked, were short, and contained small vesicles indicating disorder of the PRCs (Figure 3F). The outer nuclear layer, which consists of the nuclei of the PRCs, exhibited apoptotic morphology, such as chromatin condensation of the nuclei (Figure 3G), and many vacuoles of different sizes in Tg(rho:hsa.HTRA1) (Figure 3, F and H). The unhealthy condition of the RPE or PRCs, resulting from incomplete turnover of photoreceptor disks, leads to lipofuscin storage bodies. ${ }^{43,44}$

An autofluorescent image of retinal cryosections was examined to detect accumulation of lipofuscins (Supplemental Figure S2). Lipofuscin fluorescence in the human retina was studied noninvasively by spectrophotometry and imaging. ${ }^{44,45}$ It is known that increased autofluorescence can precede or coexist with the earliest stage of pathology in AMD. ${ }^{45,46}$ An increase in autofluorescence was seen between the outer segments of the PRCs and the RPE layer in $T g$ (rho:hsa.HTRAl) compared with WT.

These findings indicate that an increase in HTRA1 of the PRCs can induce an early AMD-like phenotype with features of the accumulation of lipofuscin.

\section{HTRA1 Overexpression Causes PRC Death}

Because of the absence of viable and healthy RPE or PRCs in $T g$ (rho:hsa.HTRAl), the function of HTRAl was studied in PRCs. The numbers of EGFP-labeled rod PRCs in $T g$ (rho:hsa.HTRAl) and WT zebrafish, which were interbred with the fish line expressing EGFP specific for rod PRCs, were examined (Figure 4A). The number of PRCs with $T g$ (rho:hsa.HTRA1) started to decrease significantly at $5 \mathrm{dpf}$ (Figure 4B). The increase in the number of PRCs from 3 to $7 \mathrm{dpf}$ in WT and rho::HTRAl fish was as follows: $31.4 \pm 4.1$ (WT) and $28.9 \pm 5.8[\mathrm{Tg}($ rho:hsa.HTRAl)] at 3 $\operatorname{dpf}(P=0.838), 38.7 \pm 4.0(\mathrm{WT})$ and $37.4 \pm 5.4$ [Tg(rho:hsa.HTRA1)] at $4 \mathrm{dpf}(P=0.510), 50.4 \pm 3.9$ (WT) and $24.8 \pm 6.4[$ Tg(rho:hsa.HTRAl) $]$ at $5 \mathrm{dpf}$ $(P<0.0001), \quad 56.4 \pm 3.6(\mathrm{WT})$ and $19.3 \pm 6.1$ $[$ Tg(rho:hsa.HTRA1)] at $6 \mathrm{dpf}(P<0.0001)$, and $61.5 \pm 5.3$ (WT) and $13.0 \pm 4.3[$ Tg(rho:hsa.HTRAl) $]$ at $7 \mathrm{dpf}$ $(P<0.0001)$.

Using the TUNEL assay and quantitative PCR of apoptosis-associated caspases, it was confirmed that the reduction of rod PRCs was caused by PRC death (Figure 4, $\mathrm{C}-\mathrm{E})$. In the TUNEL assay, a few TUNEL-positive cells were detected only in the PRC layer of $T g$ (rho:hsa.HTRAl). The number of TUNEL-positive cells in the PRC layer was significantly increased in $T g$ (rho:hsa.HTRAl)l compared with WT $[0.1 \pm 0.3$ cells per slide in WT versus $4.3 \pm 1.8$ cells per slide in Tg(rho:hsa.HTRA1); $P=0.0023$ ] (Figure 4D). The mRNA levels of caspase 3, apoptosisrelated cysteine peptidase a $(\operatorname{casp} 3 a)$, and caspase $3 b$
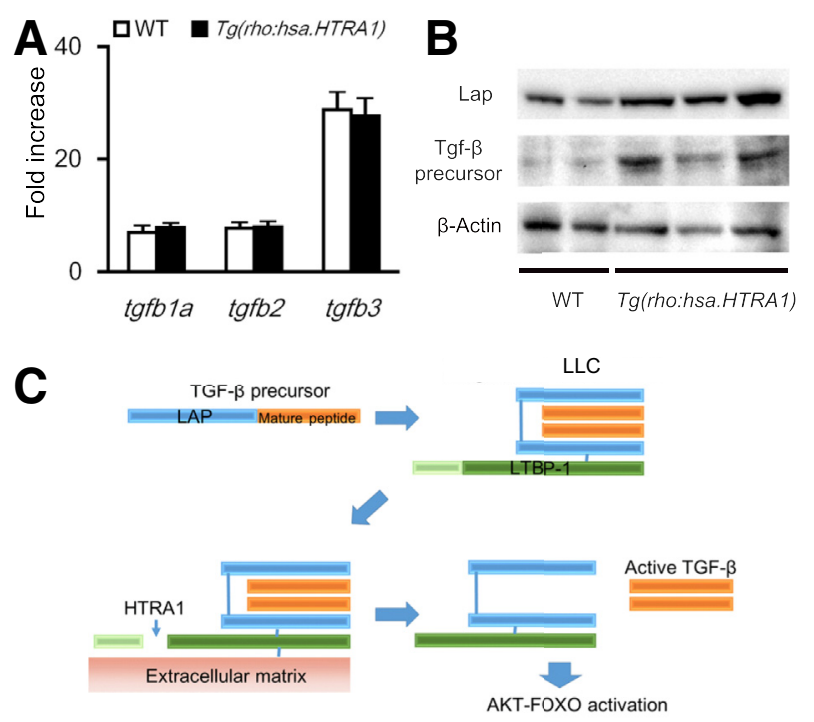

D
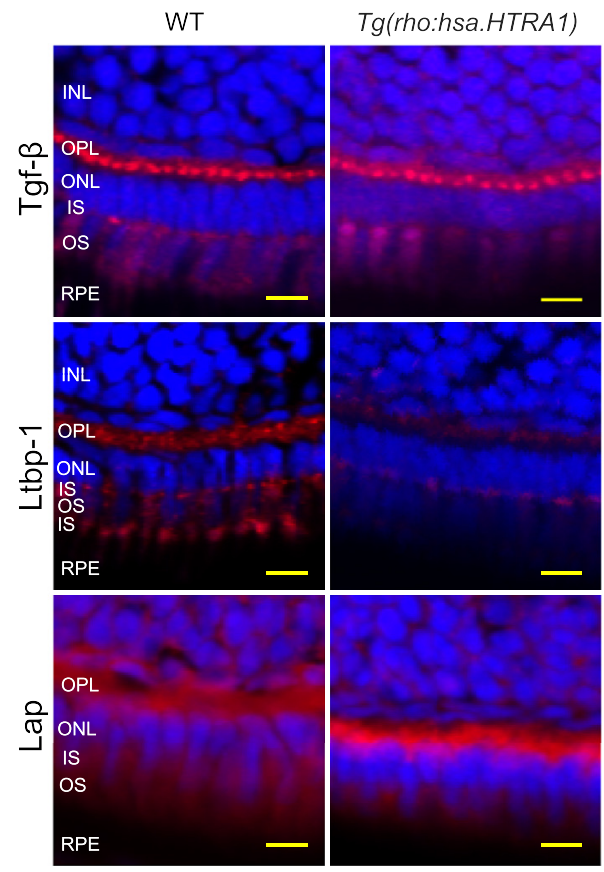

Figure 6 HTRA1 dissolves components of the large latent complex (LLC) and facilitates activation of transforming growth factor (Tgf)- $\beta$. A: Expression of mRNA of TGF- $\beta$ isoforms (tgfb1a, tgfb2, and tgfb3) in wild-type (WT) zebrafish and $T g$ (rho:hsa.HTRA1) at 5 days post-fertilization (dpf). Data are normalized to $a c t b 1$. B: Immunoblotting of Tgf- $\beta$ and latency-associated peptide (Lap) in wild-type (WT) zebrafish and $T g$ (rho:hsa.HTRA1) at $5 \mathrm{dpf}$. C: Schematic diagram of interaction between HTRA1 and LLC. HTRA1 facilitates activation of TGF- $\beta$ under disease conditions by degrading latent TGF- $\beta$ bp1 (LTBP-1). D: Immunohistochemistry of Tgf- $\beta$, Ltbp-1, and Lap in retinal sections of WT zebrafish and Tg(rho:hsa.HTRA1) at $5 \mathrm{dpf}$. Target proteins (Tgf- $\beta$, Ltbp-1, and Lap) are visualized in red. Nuclei (blue) are counterstained by DAPI. Scale bar $=5 \mu \mathrm{m}$ (D). FOX0, forkhead box 0; INL, inner nuclear layer; IS, inner segment; $0 \mathrm{NL}$, outer nuclear layer; $\mathrm{OPL}$, outer plexiform layer; $0 \mathrm{~S}$, outer segment; RPE, retinal pigment epithelium.

(casp3b), which are human caspase 3 paralogs in zebrafish, as well as caspase 8 (casp8) were also examined (Figure 4E). The mRNA levels of casp $3 a$ and casp 8 increased significantly, although that of casp $3 b$ did not 
A

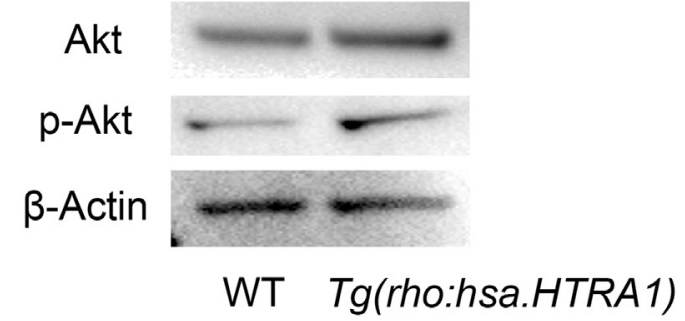

B

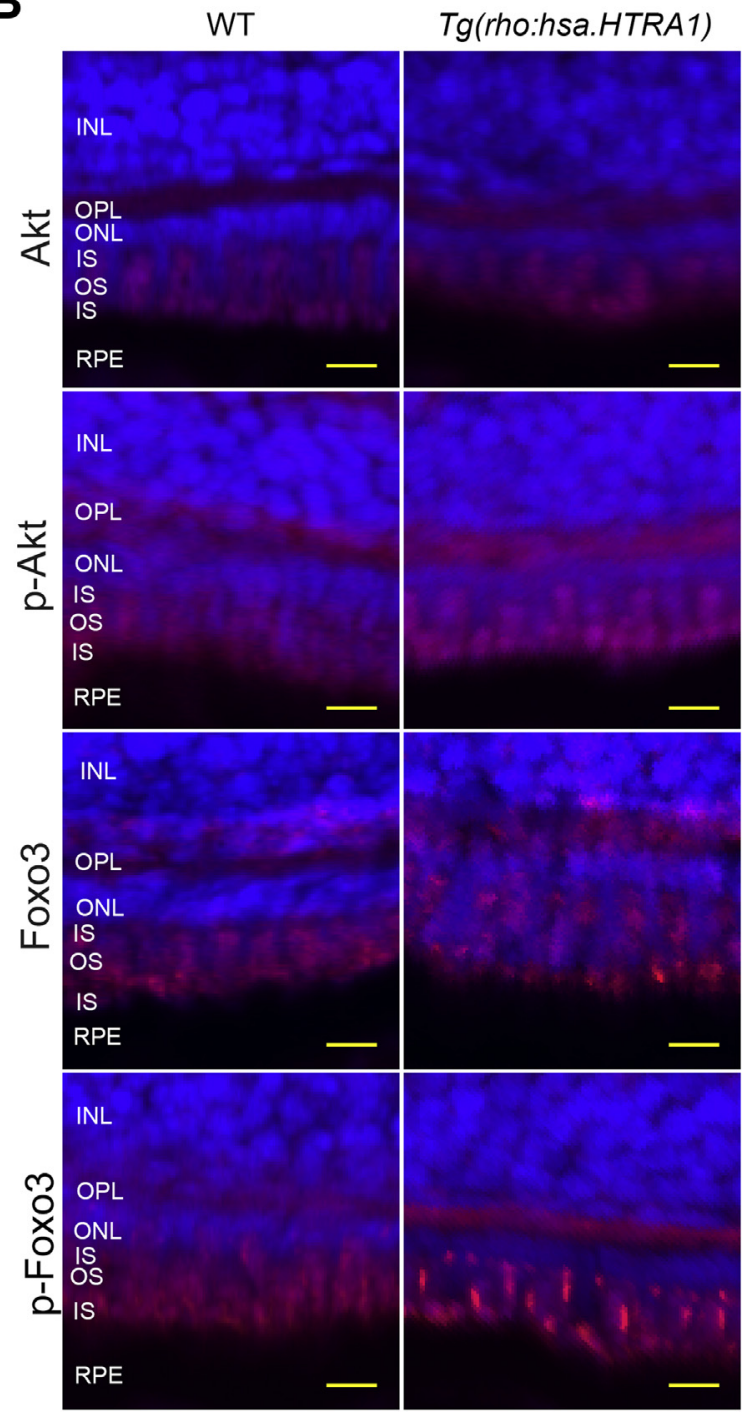

Figure 7 Overexpression of HTRA1 activates the transforming growth factor (TGF)- $\beta$-AKT-forkhead box 0 (FOXO) 3 signaling pathway. A: Immunoblotting of Akt and phosphorylated Akt (p-Akt) in wild-type (WT) zebrafish and Tg(rho:hsa.HTRA1) at 5 days post-fertilization (dpf). B: Immunohistochemistry of Akt, p-Akt, Foxo3a, and phosphorylated Foxo3 (p-Foxo3a) in retinal sections of WT zebrafish and Tg(rho:hsa.HTRA1) at $5 \mathrm{dpf}$. Target proteins (Tgf- $\beta$, latent TGF- $\beta$ bp1, latency-associated peptide, Akt, p-Akt, Foxo3, and p-Foxo3) are visualized in red. Nuclei (blue) are counterstained by DAPI. Scale bar $=5 \mu \mathrm{m}$ (B). INL, inner nuclear layer; IS, inner segment; ONL, outer nuclear layer; OPL, outer plexiform layer; $0 \mathrm{~S}$, outer segment; RPE, retinal pigment epithelium.

change. An HTRA1 inhibitor (6-boroV; DPMFKLboroV) ${ }^{38}$ was injected into the yolk of 5-dpf embryos of $T g(r h o: h$ sa.HTRA1) to confirm that HTRA1 caused PRC death (Figure 4, F and G). The reduction of PRC number was suppressed 24 hours after injection of 6-boroV in $T g$ (rho:hsa.HTRAl) (Figure 4F), and significant recovery of the number of rod PRCs was obtained at control versus $100 \mathrm{mmol} / \mathrm{L}$ and control versus $300 \mathrm{mmol} / \mathrm{L}$, with recovery rates of approximately $25.0 \%$ (control with $100 \mathrm{mmol} / \mathrm{L}$ ) and $27.7 \%$ (control with $300 \mathrm{mmol} / \mathrm{L}$ ) (Figure $4 \mathrm{G}$ ).

This analysis showed that PRC-specific overexpression of HTRA1 induced PRC death.

\section{HTRA1 Expression in an RP Model}

Apoptosis of PRCs induced by cell-specific overexpression of HTRAl suggested that HTRAl has an important role in the survival of PRCs. For further confirmation, a photoreceptor degeneration model of transgenic fish overexpressing human rhodopsin with the point mutation of rhodopsin Q344ter [Tg(rho:hsa.RHO_Q344ter)] was studied (Figure 5A). The Q344ter mutation is widely known as a pathogenic variation typical of human RP. In immunohistochemical images of 5-dpf and 2-mpf $T g(r h o: h s a . R-$ HO_Q344ter) fish, some granular positive stains of htra1 were detected in the inner segmental layer of the PRCs (Figure 5A). In addition, the level of htrala mRNA was significantly increased in the degenerated retina of Tg(rho:hsa.RHO_Q344ter) compared with WT at $5 \mathrm{dpf}$, although the level of htralb mRNA was not changed (Figure 5B). In summary, the expression of htrala was increased in PRCs of the photoreceptor degeneration model.

\section{Suppression of HTRA1 Rescues PRCs from Death}

For further confirmation of the function of HTRAl in PRC death, the HTRA1 in the photoreceptor degeneration model was prevented by rescuing PRCs from death by injecting the HTRA1 inhibitor 6-boroV into Tg(rho:hsa.RHO_Q344ter), as described above for $T g$ (rho:hsa.HTRAl) (Figure 4, F and G). The HTRA1 inhibitor rescued approximately $43.9 \%$ of PRCs from death 24 hours after injection (Figure 5, C and D). For further confirmation of the association of HTRAl with PRC death, htrala was knocked down using morpholino antisense oligonucleotides (Figure 5, E and F); $32.3 \%$ recovery of PRC numbers was observed in $T g$ (rho:hsa.RHO_Q344ter) at $5 \mathrm{dpf}$ with preventive treatment compared with nontreatment.

From these results, it can be concluded that HTRAl has an important role in the maintenance of PRCs and that overexpression of HTRAl can cause PRC death.

\section{HTRA1 Up-Regulates the TGF- $\beta$-AKT Signaling Cascade in the Process of PRC Death}

Overexpression of HTRAl phenotypically induced PRC death; however, the detailed mechanism of PRC death remained unclear. Therefore, the influence of overexpression of HTRAl on TGF- $\beta$ signaling was studied in the zebrafish retina (Figures 6 and 7). The expression levels of 
zebrafish Tgf- $\beta$ ( $t g f b)$ isoforms in Tg(rho:hsa.HTRAl) and WT fish at $5 \mathrm{dpf}$ were compared by real-time PCR and immunoblotting (Figure 6, A and B). No differences were found among $t g f b$ isoforms ( $\operatorname{tg} f b l a$, $\operatorname{tg} f b 2$, and $\operatorname{tg} f b 3)$ at the mRNA level (Figure 6A), but at the protein level, Tgf- $\beta$ precursor and LAP were increased in Tg(rho:hsa.HTRAl) fish (Figure 6B). Herein, we explain the mechanism of maturation of TGF- $\beta$ mediated by HTRA1 (Figure 6C). LAP is a by-product containing the $\mathrm{N}$-terminal region of the TGF- $\beta$ gene product in the processing of mature TGF- $\beta .^{47}$ TGF- $\beta$ forms a complex called the large latent complex, consisting of a homodimer of TGF- $\beta$, LAP, and LTBP- $1 .{ }^{48}$ After large latent complex is secreted from the cell, it binds to the extracellular matrix via LTBP-1 and remains there in an inactive state. LTBP-1 and LAP need to be disassembled for TGF- $\beta$ to change into an active state, and HTRA1 is one of the serine proteases that process both LAP and LTBP-1. However, it is unclear how HTRA1 affects the activation of TGF- $\beta$ signaling in diseased conditions in vivo by proteolysis of LAP and LTBP-1. The existence and location of Tgf- $\beta$, Lap, and Ltbp- 1 in the outer retinal layer of $T g$ (rho:hsa.HTRAl) and WT fish were studied at $5 \mathrm{dpf}$ by immunohistochemistry (Figure 6D). Tgf- $\beta$ was localized in the outer plexiform layer and the inner segment of the PRCs. Unlike the findings from immunoblotting, with the use of immunohistochemistry, no difference was found between $T g$ (rho:hsa.HTRAl) and WT in the expression of Tgf- $\beta$, except for diffuse light stains of the nucleus and cytoplasm. However, Ltbp-1 was found to be decreased in the outer plexiform layer and the inner segment of the PRCs and, in contrast, lap was increased in the outer nuclear layer in $T g$ (rho:hsa.HTRAl) (Figure 6D). The previous report showed that HTRA1 dissected LTBP-1 attaching large latent complex on the extracellular matrix and increased activated free TGF- $\beta{ }^{49}$ The lack of the signal of Ltbp- 1 and the increase in Lap indicated that Tgf- $\beta$ signaling was activated.

For further confirmation of the association of TGF- $\beta$ signaling with PRC death induced by HTRAl, the downstream activation of TGF- $\beta$ was examined. Among many cascades, such as the SMAD pathway and the non-SMAD pathway, including TGF- $\beta$ activated kinase $1-$ mitogenactivated protein kinase, Rho-Rho-associated coiled-coilcontaining protein kinase, and AKT-mammalian target of rapamycin, the activation of TGF- $\beta-$ AKT-FOXO signaling was studied. It was because phosphatidylinositol 3-kinase/ AKT is modulated by TGF- $\beta$ and regulates processes of cell survival and apoptosis, ${ }^{50}$ and resveratrol, which activates FOXO via sirtuin 1, may have the potential to prevent the progression of AMD (Figure 7). ${ }^{51}$ AKT phosphorylates FOXO and exports it out of the nucleus. In other words, AKT blocks FOXO transcription of essential genes for cell survival, such as antistress, apoptosis, and DNA damage response. ${ }^{52,53}$

The phosphorylation of Akt in Tg(rho:hsa.HTRAl) was analyzed by immunoblotting and immunohistochemistry (Figure 7). Immunoblotting of the whole eye of 5-dpf
Tg(rho:hsa.HTRAl) fish showed that both Akt and p-Akt were increased to a small extent (Figure 7A). To detect in which retinal layers Akt and p-Akt were increased, immunohistochemistry of these molecules was performed in zebrafish. As expected, p-Akt was slightly increased in the outer plexiform layer and inner segment of the PRCs, although there was no difference in Akt levels between Tg(rho:hsa.HTRA1) and WT eyes (Figure 7B). foxo3, which is a zebrafish ortholog gene of $\mathrm{FOXO3}$ located downstream of p-Akt, was also increased, and the levels of phosphorylation were higher in $T g$ (rho:hsa.HTRAl) than in WT eyes (Figure 7B). The expression levels of Foxo3 were increased in every outer retinal layer involving the intranucleus of the PRCs, and phosphorylated Foxo3 showed elongated strong stains among weak stains of the inner segments as a consequence of the disappearance of Foxo3 from the nucleus (Figure 7B). These results indicate that HTRA1 increases activated Tgf- $\beta$ by disassembling Ltbp- 1 and promotes phosphorylation of Akt and Foxo3. Enhanced phosphorylation of FOXO3 inhibits the transcriptional activity of $\mathrm{FOXO3} .^{54}$

\section{Discussion}

The present study supports two important findings of the pathogenesis of early AMD. The first finding is that PRCs, as well as the RPE, express HTRAl, but contrary to HTRAl expression by the RPE, HTRAl expression in PRCs is increased by aging and genetic background. The second finding is that increased expression of HTRAl by rod PRCs can induce apoptosis of PRCs and morphologic changes of the retina resembling those in early AMD. Even in animal models, these findings strongly suggest not only that AMD is a disorder of the RPE, but that PRC death is one of the primary events in the progress of AMD.

To the best of our knowledge, this is the first report showing that PRCs increase the expression of Htral aging, except for the AMD model. Analysis of the expression of htral clearly showed an increase with aging. Previous studies reported detection of HTRAl in the RPE, Bruch membrane, and choroid of eyes from normal elderly men. ${ }^{29}$ However, there has been no assessment of changes in HTRAl with aging. Aging is thought to have the most important role in the pathogenesis of AMD. ${ }^{55}$ Thus, increased expression of htral in PRCs is an important finding. A disease-associated SNP in the HTRAl promoter region increased HTRAl expression in PRCs more than in the RPE, as shown herein. A recent study reported the same effect of the HTRAl promoter region in PRCs. ${ }^{56}$ These findings give us a new insight that aging- and diseaseassociated mutations are important risk factors for AMD, because a continuing expression of HTRAl from PRCs gradually produces degeneration of both the RPE and PRCs over a long period. 
HTRAl was found to be associated with the maintenance of PRCs. Overexpression of HTRAl in rod PRCs induces apoptosis of PRCs. Death of PRCs is shown to be a specific effect of HTRAl, because the cells are rescued by an HTRA1 inhibitor. Moreover, increased expression of htral was found in the retina of Tg(rho:hsa.RHO_Q344ter), which carries a mutation that causes RP, a human photoreceptor degenerative disease. This zebrafish model of RP shows strong apoptosis of PRCs, a phenotype that is rescued by an HTRA1 inhibitor or htrala knockdown. This finding indicates that induction of HTRAl may have an important role in the degeneration of PRCs and may universally have a harmful effect on the homeostatic properties of PRCs in pathologic conditions.

Recent reports of HTRAl in AMD have focused mainly on functional analyses of the RPE and have shown that overexpression of HTRAl in the RPE of transgenic mice induces conformational changes in both the RPE and the choroid, with neovascularization, such as polypoidal CNV as a subtype of AMD. ${ }^{29,57}$ Vierkotten et $\mathrm{al}^{58}$ also referred to the degeneration of PRCs, but it remained unclear whether the degeneration of PRCs was directly induced by HTRAI or occurred subsequently to degeneration of the RPE. Herein, it was demonstrated that an increase in endogenous HTRAl expressed by PRCs directly induced PRC death without degeneration of other retinal layers, including the RPE.

In the PRC degeneration model, overexpression of HTRAl induces accumulation of lipofuscin, an early AMDlike hallmark. It is suggested that initial changes of AMD, such as reticular pseudodrusen (RPDs) and hyperreflective foci, are associated with HTRAl rs $11201638 .{ }^{59,60}$ In this model, lipofuscin accumulates between the outer segment of the PRCs and the apical side of the RPE. The accumulation of lipofuscin is different from the accumulation of common drusen at the suprabasal side of the RPE, but it resembles the accumulation of lipofuscin seen in RPDs. RPDs, as well as common drusen, are recognized as the hallmark of AMD and are strong predictors of progression to both geographic atrophy and neovascular AMD. ${ }^{59,61,62}$ RPDs consist of aggregates of microglia and lipofuscin and are frequently observed in patients with genetic aberrations of HTRAl. ${ }^{61}$ No previous model of AMD, including the transgenic mouse overexpressing HtrA1 in the RPE, showed accumulation of lipofuscin similar to RPDs. Therefore, we maintain that the transgenic fish overexpressing HTRAl in PRCs are the first model that fairly replicates the features of early AMD.

Further analysis of the phenotype of $T g$ (rho:hsa.HTRAl) revealed that enhanced HTRAl in PRCs activates the TGF- $\beta$ signaling pathway. HTRA1 causes degeneration of Ltbp-1 and increases Tgf- $\beta$ precursor in the outer retina, according to immunohistochemistry and Western blot analysis. The data show that degradation of Ltbp-1 induced by HTRA1 results in activation of TGF- $\beta$ signaling in the transgenic zebrafish retina. In essence, HTRAl behaves in vivo not only as a protease, disrupting construction of retinal layers, but also as a signal transducer of TGF- $\beta$ in PRCs. Also, htrala expression was increased in PRCs in the RP model. Considering that the previous report showed that an increase in HTRA1 was correlated with an increase in vascular endothelial growth factor levels with retinal detachment, ${ }^{63}$ this finding indicates that up-regulation of TGF- $\beta$ causes an increase in HTRA1 in the RP in the same way as in other PRC-impairing diseases. In summary, expression of HTRAl triggers not only AMD but any other disease involving degeneration of PRCs, and therefore, any neuroprotective therapy targeting HTRAl is potentially suitable for the treatment of retinal degenerative disease.

The present study demonstrated that the TGF$\beta-\mathrm{AKT}-\mathrm{FOXO}$ pathway, as one of several signaling cascades of TGF- $\beta$, induces apoptosis of PRCs. In AMD, resveratrol, which is a stilbenoid, accelerates the transcription activity of FOXOs via sirtuin 1 and prevents the development of AMD by anti-inflammatory and antioxidant effects. ${ }^{51}$ A recent study showed that, in the experimental rat model of retinal detachment, resveratrol induced an increase in FOXOs and consequently prevented apoptosis of PRCs. ${ }^{64}$ The FOXO family was, therefore, studied. Among FOXO isoforms, foxo3a (foxo3 in zebrafish) is thought to be one of the prospective antiapoptotic targets. This is because FOXO3 is essential for resistance to oxidative stress, and deletion of FOXO3 leads to depletion of reactive oxygen species-scavenging enzymes and increase in reactive oxygen species. ${ }^{65,66}$ Oxidative stress is closely associated with AMD, as shown by epidemiologic studies that found that smoking, which is known to cause oxidative damage and impair RPE function, is one of the major risk factors for AMD. ${ }^{67}$ FOXO3 is likely one of the transcriptional factors associated with TGF- $\beta$-induced apoptosis of PRCs associated with HTRAl.

It has been controversial as to which gene of the ARMS2/HTRA1 region is responsible for the pathogenesis of AMD. ${ }^{23,68-70}$ A genetic study reported that the ARMS2 locus was more closely associated with AMD than was the HTRA1 locus. The mechanisms by which these loci affect AMD development are not clear. It is possible that the ARMS2 locus also has some positional effects on HTRAl expression. Other AMD-associated HTRAl variants have a role in the regulation of TGF- $\beta$ signaling. ${ }^{71}$ Combined with these experiments, HTRAl effects on TGF- $\beta$ signaling may have a role in the development of AMD.

In conclusion, HTRA1 derived from rod PRCs can induce apoptosis of PRCs and morphologic changes in the retina like those in early AMD. From the viewpoint of HTRAl, AMD originates from disturbance of PRCs before degeneration of the RPE. AMD is initially a disease of the PRCs. HTRA1 derived from rod PRCs is associated with various PRC degenerative diseases, including early AMD, and therefore HTRAI is a potential target of neuroprotective therapies for early AMD and other degenerative diseases of the PRCs. 


\section{Acknowledgments}

The authors thank the staff of Daiichi-Sankyo Co, Ltd, for helpful support with transmission electron microscopy and in situ hybridization and provision of high-temperature requirement A 1 inhibitor; Takeshi Nakao (Osaka University) for helpful advice about experimental procedures for zebrafish; and Dr. Shoji Kawamura (The University of Tokyo) for providing $T g(r h o: E G F P)$.

M.T. had full access to all the data in the study and had final responsibility for the decision to submit for publication.

\section{Supplemental Data}

Supplemental material for this article can be found at https://doi.org/10.1016/j.ajpath.2018.08.012.

\section{References}

1. Curcio CA, Medeiros NE, Millican CL: Photoreceptor loss in agerelated macular degeneration. Invest Ophthalmol Vis Sci 1996, 37: $1236-1249$

2. Klein R, Lee KE, Gangnon RE, Klein BE: Incidence of visual impairment over a 20-year period: the Beaver Dam eye study. Ophthalmology 2013, 120:1210-1219

3. Swaroop A, Chew EY, Rickman CB, Abecasis GR: Unraveling a multifactorial late-onset disease: from genetic susceptibility to disease mechanisms for age-related macular degeneration. Annu Rev Genomics Hum Genet 2009, 10:19-43

4. Din X, Patel M, Chan CC: Molecular pathology of age-related macular degeneration. Prog Retin Eye Res 2009, 28:1-18

5. Wu Z, Ayton LN, Luu CD, Guymer RH: Relationship between retinal microstructures on optical coherence tomography and microperimetry in age-related macular degeneration. Ophthalmology 2014, 121: $1445-1452$

6. Yang S, Zuo C, Xiao H, Mi L, Luo G, Xu X, Liu X: Photoreceptor dysfunction in early and intermediate age-related macular degeneration assessed with mfERG and spectral domain OCT. Doc Ophthalmol 2016, 132:17-26

7. Takahashi A, Ooto S, Yamashiro K, Oishi A, Tamura H, Nakanishi H, Ueda-Arakawa N, Tsujikawa A, Yoshimura N: Photoreceptor damage and reduction of retinal sensitivity surrounding geographic atrophy in age-related macular degeneration. Am J Ophthalmol 2016, 168:260-268

8. Gibson JM, Gibson SJ: A safety evaluation of ranibizumab in the treatment of age-related macular degeneration. Expert Opin Drug Saf 2014, 13:1259-1270

9. Cunningham ET Jr, Feiner L, Chung C, Tuomi L, Ehrlich JS: Incidence of retinal pigment epithelial tears after intravitreal ranibizumab injection for neovascular age-related macular degeneration. Ophthalmology 2011, 118:2447-2452

10. Goldstein M, Heilweil G, Barak A, Loewenstein A: Retinal pigment epithelial tear following photodynamic therapy for choroidal neovascularization secondary to AMD. Eye 2015, 19:1315-1324

11. Comparison of Age-Related Macular Degeneration Treatments Trials (CATT) Research Group, Maguire MG, Martin DF, Ying GS, Jaffe GJ, Daniel E, Grunwald JE, Toth CA, Ferris FL 3rd, Fine SL: Five-year outcomes with anti-vascular endothelial growth factor treatment of neovascular age-related macular degeneration: the comparison of age-related macular degeneration treatments trials. Ophthalmology 2015, 123:1751-1756
12. Finger RP, Guymer RH, Gillies MC, Keeffe JE: The impact of antivascular endothelial growth factor treatment on quality of life in neovascular age-related macular degeneration. Ophthalmology 2014, $21: 1246-1251$

13. Chakravarthy U, Wong TY, Fletcher A, Piault E, Evans C, Zlateva G, Buggage R, Pleil A, Mitchell P: Clinical risk factors for age-related macular degeneration: a systematic review and meta-analysis. BMC Ophthalmol 2010, 10:31

14. Thornton J, Edwards R, Mitchell P, Harrison RA, Buchan I, Kelly SP: Smoking and age-related macular degeneration: a review of association. Eye 2005, 19:935-944

15. Clemons TE, Milton RC, Klien R, Seddon JM, Ferris FL III; AgeRelated Eye Disease Study Research Group: Risk factors for the incidence of advanced age-related macular degeneration in the AgeRelated Eye Disease Study (AREDS) AREDS report no. 19. Ophthalmology 2005, 112:533-539

16. Shen JK, Dong A, Hackett SF, Bell WR, Green WR, Campochiaro PA: Oxidative damage in age-related macular degeneration. Histol Histopathol 2007, 22:1301-1308

17. Baird PN, Robman LD, Richardson AJ, Dimitrov PN, Tikellis G, McCarty CA, Guymer RH: Gene-environment interaction in progression of AMD: the $\mathrm{CFH}$ gene, smoking and exposure to chronic infection. Hum Mol Genet 2008, 17:1299-1305

18. Fritsche LG, Chen W, Schu M, Yaspan BL, Yu Y, Thorleifsson G, et al: Seven new loci associated with age-related macular degeneration. Nat Genet 2013, 45:433-439. 439e1-439e2

19. Jakobsdottir J, Conley YP, Weeks DE, Mah TS, Ferrell RE, Gorin MB: Susceptibility genes for age-related maculopathy on chromosome 10q26. Am J Hum Genet 2005, 77:389-407

20. Fisher SA, Abecasis GR, Yashar BM, Zareparsi S, Swaroop A, Iyengar SK, Klein BE, Klein R, Lee KE, Majewski J, Schultz DW, Klein ML, Seddon JM, Santangelo SL, Weeks DE, Conley YP, Mah TS, Schmidt S, Haines JL, Pericak-Vance MA, Gorin MB, Schulz HL, Pardi F, Lewis CM, Weber BH: Meta-analysis of genome scans of agerelated macular degeneration. Hum Mol Genet 2005, 14:2257-2264

21. Fritsche LG, Igl W, Bailey JN, Grassmann F, Sengupta S, BraggGresham JL, et al: A large genome-wide association study of agerelated macular degeneration highlights contributions of rare and common variants. Nat Genet 2016, 48:134-143

22. Dewan A, Liu M, Hartman S, Zhang SS, Liu DT, Zhao C, Tam PO, Chan WM, Lam DS, Snyder M, Barnstable C, Pang CP, Hoh J: HTRA1 promoter polymorphism in wet age-related macular degeneration. Science 2006, 314:989-992

23. Yang Z, Camp NJ, Sun H, Tong Z, Gibbs D, Cameron DJ, Chen H, Zhao Y, Pearson E, Li X, Chien J, Dewan A, Harmon J, Bernstein PS, Shridhar V, Zabriskie NA, Hoh J, Howes K, Zhang K: A variant of the HTRA1 gene increases susceptibility to age-related macular degeneration. Science 2006, 314:992-993

24. Grassmann F, Heid IM, Weber BH; International AMD Genomics Consortium (IAMDGC): Recombinant haplotypes narrow the ARMS2/HTRA1 association signal for age-related macular degeneration. Genetics 2017, 205:919-924

25. Hara K, Shiga A, Fukutake T, Nozaki H, Miyashita A, Yokoseki A, Kawata H, Koyama A, Arima K, Takahashi T, Ikeda M, Shiota H, Tamura M, Shimoe Y, Hirayama M, Arisato T, Yanagawa S, Tanaka A, Nakano I, Ikeda S, Yoshida Y, Yamamoto T, Ikeuchi T, Kuwano R, Nishizawa M, Tsuji S, Onodera O: Association of HTRA1 mutations and familial ischemic cerebral small-vessel disease. N Engl J Med 2009, 360:1729-1739

26. Hou Y, Lin H, Zhu L, Liu Z, Hu F, Shi J, Yang T, Shi X, Guo H, Tan X, Zhang L, Wang Q, Li Z, Zhao Y: The inhibitory effect of IFN$\gamma$ on protease HTRA1 expression in rheumatoid arthritis. J Immunol 2014, 193:130-138

27. Grau S, Richards PJ, Kerr B, Hughes C, Caterson B, Williams AS, Junker U, Jones SA, Clausen T, Ehrmann M: The role of human HtrA1 in arthritic disease. J Biol Chem 2006, 28 : $6124-6129$ 
28. Chien J, Aletti G, Baldi A, Catalano V, Muretto P, Keeney GL, Kalli KR, Staub J, Ehrmann M, Cliby WA, Lee YK, Bible KC, Hartmann LC, Kaufmann SH, Shridhar V: Serine protease HtrA1 modulates chemotherapy-induced cytotoxicity. J Clin Invest 2006, 116:1994-2004

29. Jones A, Kumar S, Zhang N, Tong Z, Yang JH, Watt C, Anderson J, Amrita, Fillerup H, McCloskey M, Luo L, Yang Z, Ambati B, Marc R, Oka C, Zhang K, Fu Y: Increased expression of multifunctional serine protease, HTRA1, in retinal pigment epithelium induces polypoidal choroidal vasculopathy in mice. Proc Natl Acad Sci U S A 2011, 108:14578-14583

30. Kimmel CB, Ballard WW, Kimmel SR, Ullmann B, Schilling TF: Stages of embryonic development of the zebrafish. Dev Dyn 1995, 203:253-310

31. Malicki J, Jo H, Wei X, Hsiung M, Pujic Z: Analysis of gene function in the zebrafish retina. Methods 2002, 28:427-438

32. Nakao T, Tsujikawa M, Notomi S, Ikeda Y, Nishida K: The role of mislocalized phototransduction in photoreceptor cell death of retinitis pigmentosa. PLoS One 2012, 7:e32472

33. Hamaoka T, Takechi M, Chinen A, Nishiwaki Y, Kawamura S: Visualization of rod photoreceptor development using GFPtransgenic zebrafish. Genesis 2002, 34:215-220

34. Truebestein L, Tennstaedt A, Mönig T, Krojer T, Canellas F, Kaiser M, Clausen T, Ehrmann M: Substrate-induced remodeling of the active site regulates human HTRA1 activity. Nat Struct Mol Biol 2011, 18:386-388

35. Latendresse JR, Warbrittion AR, Jonassen H, Creasy DM: Fixation of testes and eyes using a modified Davidson's fluid: comparison with Bouin's fluid and conventional Davidson's fluid. Toxicol Pathol 2002, 30:524-533

36. Delori F, Keilhauer C, Sparrow JR, Staurenghi G: Origin of fundus autofluorescence. Atlas of Fundus Autofluorescence Imaging. Edited by Holz F, Spaide R, Bird AC, Schmitz-Valckenberg S. Berlin, Germany: Springer, 2007. pp. 17-29

37. Flynn E, Ueda K, Auran E, Sullivan JM, Sparrow JR: Fundus autofluorescence and photoreceptor cell rosettes in mouse models. Invest Ophthalmol Vis Sci 2014, 55:5643-5652

38. Kim GY, Kim HY, Kim HT, Moon JM, Kim CH, Kang S, Rhim H: HtrA1 is a novel antagonist controlling fibroblast growth factor (FGF) signaling via cleavage of FGF8. Mol Cell Biol 2012, 32:4482-4492

39. Zheng W, Wang Z, Collins JE, Andrews RM, Stemple D, Gong Z: Comparative transcriptome analyses indicate molecular homology of zebrafish swimbladder and mammalian lung. PLoS One 2011, 6: e24019

40. Clausen T, Southan C, Ehrmann M: The HtrA family of proteases: implications for protein composition and cell fate. Mol Cell 2002, 10: $443-455$

41. Lana TP, da Silva Costa SM, Ananina G, Hirata FE, Rim PHH, Medina FM, de Vasconcellos JPC, de Melo MB: Association of HTRA1 rs11200638 with age-related macular degeneration (AMD) in Brazilian patients. Ophthalmic Genet 2018, 39:46-50

42. Ruamviboonsuk P, Tadarati M, Singhanetr P, Wattanapokayakit S, Kunhapan P, Wanitchanon T, Wichukchinda N, Mushiroda T, Akiyama M, Momozawa Y, Kubo M, Mahasirimongkol S: Genomewide association study of neovascular age-related macular degeneration in the Thai population. J Hum Genet 2017, 62:957-962

43. Feeney L: Lipofuscin and melanin of human retinal pigment epithelium: fluorescence, enzyme cytochemical, and ultrastructural studies. Invest Ophthalmol Vis Sci 1978, 17:583-600

44. Kevany BM, Palczewski K: Phagocytosis of retinal rod and cone photoreceptors. Physiology 2010, 25:8-15

45. Arend O, Weiter JJ, Goger D, Delori FC: Lipofuscin and drusen fluorescence in aging and age-related macular degeneration. Ophthalmologe 1995, 92:647-653

46. Delori FC, Fleckner MR, Goger DG, Weiter JJ, Dorey CK: Autofluorescence distribution associated with drusen in age-related macular degeneration. Invest Ophthalmol Vis Sci 2000, 41:496-504
47. Rifkin DB: Latent transforming growth factor- $\beta$ (TGF- $\beta$ ) binding proteins: orchestrators of TGF- $\beta$ availability. J Biol Chem 2003, 280: $7409-7412$

48. Kanzaki T, Olofsson A, Morén A, Wernstedt C, Hellman U, Miyazono K, Claesson-Welsh L, Heldin CH: TGF-beta 1 binding protein: a component of the large latent complex of TGF-beta 1 with multiple repeat sequences. Cell 1990, 61:1051-1061

49. Beaufort N, Scharrer E, Kremmer E, Lux V, Ehrmann M, Huber R, Houlden H, Werring D, Haffner C, Dichgans M: Cerebral small vessel disease-related protease HtrA1 processes latent TGF- $\beta$ binding protein 1 and facilitates TGF- $\beta$ signaling. Proc Natl Acad Sci U S A 2014, 111:16496-16501

50. Nicholson KM, Anderson NG: The protein kinase B/Akt signalling pathway in human malignancy. Cell Signal 2002, 14:381-395

51. Nagai N, Kubota S, Tsubota K, Ozawa Y: Resveratrol prevents the development of choroidal neovascularization by modulating AMPactivated protein kinase in macrophages and other cell types. J Nutr Biochem 2014, 25:1218-1225

52. Manning BD, Cantley LC: AKT/PKB signaling: navigating downstream. Cell 2007, 129:1261-1274

53. Castellano E, Downward J: RAS interaction with PI3K: more than just another effector pathway. Genes Cancer 2011, 2: $261-274$

54. Brunet A, Bonni A, Zigmond MJ, Lin MZ, Juo P, Hu LS, Anderson MJ, Arden KC, Blenis J, Greenberg ME: Akt promotes cell survival by phosphorylating and inhibiting a forkhead transcription factor. Cell 1999, 96:857-868

55. Age-Related Eye Disease Study Research Group: Risk factors associated with age-related macular degeneration: a case-control study in the age-related eye disease study: Age-Related Eye Disease Study Report Number 3. Ophthalmology 2000, 107:2224-2232

56. Iejima D, Itabashi T, Kawamura Y, Noda T, Yuasa S, Fukuda K, Oka C, Iwata T: HTRA1 (high temperature requirement A serine peptidase 1) gene is transcriptionally regulated by insertion/deletion nucleotides located at the $3^{\prime}$ end of the ARMS2 (age-related maculopathy susceptibility) gene in patients with age-related macular degeneration. J Biol Chem 2015, 290:2784-2797

57. Iejima D, Nakayama M, Iwata $T$ : Overexpression induces the exudative form of age-related macular degeneration. J Stem Cells 2015, 10:193-203

58. Vierkotten S, Muether PS, Fauser S: Overexpression of HTRA1 leads to ultrastructural changes in the elastic layer of Bruch's membrane via cleavage of extracellular matrix components. PLoS One 2011, 6: e22959

59. Zhou Q, Daniel E, Maguire MG, Grunwald JE, Martin ER, Martin DF, Ying GS; Comparison of Age-Related Macular Degeneration Treatments Trials Research Group: Pseudodrusen and incidence of late age-related macular degeneration in fellow eyes in the comparison of age-related macular degeneration treatments trials. Ophthalmology 2016, 123:1530-1540

60. Altay L, Slz P, Schick T, Felsch M, Hoyng CB, den Hollander AI, Langmann T, Fauser S: Association of hyperreflective foci present in early forms of age-related macular degeneration with known agerelated macular degeneration risk polymorphisms. Invest Ophthalmol Vis Sci 2016, 57:4315-4320

61. Finger RP, Chong E, McGuinness MB, Robman LD, Aung KZ, Giles G, Baird PN, Guymer RH: Reticular pseudodrusen and their association with age-related macular degeneration: the Melbourne collaborative cohort study. Ophthalmology 2016, 123: 599-608

62. Gil JQ, Marques JP, Hogg R, Rosina C, Cachulo ML, Santos A, Staurenghi G, Chakravarthy U, Silva R: Clinical features and longterm progression of reticular pseudodrusen in age-related macular degeneration: findings from a multicenter cohort. Eye 2017, 31: 364-371

63. Ng TK, Yam GH, Chen WQ, Lee VY, Chen H, Chen LJ, Choy KW, Yang Z, Pang CP: Interactive expressions of HtrA1 and VEGF in 
human vitreous humors and fetal RPE cells. Invest Ophthalmol Vis Sci 2011, 52:3706-3712

64. Huang W, Li G, Qiu J, Gonzalez P, Challa P: Protective effects of resveratrol in experimental retinal detachment. PLoS One 2013, 8:e75735

65. Marinkovic D, Zhang X, Yalcin S, Luciano JP, Brugnara C, Huber T, Ghaffari S: Foxo3 is required for the regulation of oxidative stress in erythropoiesis. J Clin Invest 2007, 117:2133-2144

66. Miyamoto K, Araki KY, Naka K, Arai F, Takubo K, Yamazaki S, Matsuoka S, Miyamoto T, Ito K, Ohmura M, Chen C, Hosokawa K, Nakauchi H, Nakayama K, Nakayama KI, Harada M, Motoyama N, Suda T, Hirao A: Foxo3a is essential for maintenance of the hematopoietic stem cell pool. Cell Stem Cell 2007, 1:101-112

67. Khan JC, Thurlby DA, Shahid H, Clayton DG, Yates JR, Bradley M, Moore AT, Bird AC; Genetic Factors in AMD Study: Smoking and age related macular degeneration: the number of pack years of cigarette smoking is a major determinant of risk for both geographic atrophy and choroidal neovascularisation. Br J Ophthalmol 2006, 90:75-80
68. Fritsche LG, Loenhardt $\mathrm{T}$, Janssen A, Fisher SA, Rivera A, Keilhauer CN, Weber BH: Age-related macular degeneration is associated with an unstable ARMS2 (LOC387715) mRNA. Nat Genet 2008, 40:892-896

69. Kanda A, Stambolian D, Chen W, Curcio CA, Abecasis GR, Swaroop A: Age-related macular degeneration-associated variants at chromosome 10q26 do not significantly alter ARMS2 and HTRA1 transcript levels in the human retina. Mol Vis 2010, 16:1317-1323

70. Friedrich U, Myers CA, Fritsche LG, Milenkovich A, Wolf A, Corbo JC, Weber BH: Risk- and non-risk-associated variants at the 10q26 AMD locus influence ARMS2 mRNA expression but exclude pathogenic effects due to protein deficiency. Hum Mol Genet 2011, 20:1387-1399

71. Friedrich U, Datta S, Schubert T, Plössl K, Schneider M, Grassmann F, Fuchshofer R, Tiefenbach KJ, Längst G, Weber BH: Synonymous variants in HTRA1 implicated in AMD susceptibility impair its capacity to regulate TGF- $\beta$ signaling. Hum Mol Genet 2015, 24:6361-6373 